\title{
Highly Diastereoselective Synthesis of Tetrahydrofurans via Lewis Acid Catalyzed Cyclopropane/Aldehyde Cycloadditions
}

\author{
Patrick D. Pohlhaus and Jeffrey S. Johnson* \\ Department of Chemistry, University of North Carolina at Chapel Hill,
}

Chapel Hill, North Carolina 27599-3290

\section{Supporting Information}

\section{Table of Contents}

$\begin{array}{ll}\text { Experimental Procedures and Spectral Data } & \text { S1-S7 }\end{array}$

Reference

Appendix (NMR Spectra)

\section{Experimental Section}

Materials and Methods: General. Proton and carbon nuclear magnetic resonance spectra $\left({ }^{1} \mathrm{H}\right.$ and ${ }^{13} \mathrm{C}$ NMR) were recorded with tetramethylsilane (TMS) as the internal standard for ${ }^{1} \mathrm{H} \mathrm{NMR}$ at $0.00 \mathrm{ppm}$ and $\mathrm{CDCl}_{3}$ solvent resonance as the internal standard for ${ }^{13} \mathrm{C}$ NMR at $77.16 \mathrm{ppm}$. ${ }^{1} \mathrm{H}$ NMR data are reported as follows: chemical shift, multiplicity $(\mathrm{s}=$ singlet, $\mathrm{d}=$ doublet, $\mathrm{t}=$ triplet, $\mathrm{q}=$ quartet, $\mathrm{sep}=$ septet, $\mathrm{m}=$ multiplet), coupling constants $(\mathrm{Hz})$, and integration. Structural assignments were made using NOESY experiments (see Appendix for spectra). Analytical thin layer chromatography (TLC) was performed on $0.25 \mathrm{~mm}$ silica gel 60 plates. Visualization was accomplished with UV light and aqueous ceric ammonium molybdate solution followed by heating. Purification of the reaction products was carried out by flash chromatography using silica gel $60(32-63 \mu \mathrm{m})$. All reactions were carried out under an atmosphere of argon in flame-dried glassware with magnetic stirring. Yield refers to isolated yield of analytically pure material. Yields are reported for a specific experiment and as a result may differ slightly from those found in the tables and equations, which are averages of at least two experiments. Methylene chloride was dried by passage through a column of neutral alumina under nitrogen prior to use. Cyclopropanes were prepared using Wood's procedure from the corresponding benzylidene malonate and trimethylsulphoxonium iodide. ${ }^{1}$ Benzylidene malonates were prepared via Knoevenagel condensations. ${ }^{1}$ Unless otherwise noted, reagents were obtained from commercial sources and used without further purification. Solid aldehydes, 4-nitrobenzaldehyde and 4-chlorobenzaldehyde were purified by sublimation prior to use. All other aldehydes were distilled from $\mathrm{CaSO}_{4}$ prior to use.

General procedure (A) for the Lewis acid catalyzed cycloaddition. In an inert atmosphere glovebox, a flame-dried vial was charged with $0.017 \mathrm{mmol}$ of $\mathrm{Sn}(\mathrm{OTf})_{2}$ (0.050 equiv), $0.34 \mathrm{mmol}$ of the cyclopropane (1.0 equiv), and a magnetic stir bar. Outside of the glove box, the vial was placed under an argon atmosphere and charged with $0.5 \mathrm{~mL}$ of $\mathrm{CH}_{2} \mathrm{Cl}_{2}$ followed by $1.0 \mathrm{mmol}$ of the aldehyde (3.0 equiv). The reaction 
was stirred at room temp $\left(23{ }^{\circ} \mathrm{C}-29^{\circ} \mathrm{C}\right)$ until the disappearance of starting material was confirmed by TLC. The reaction mixture was then passed over a plug of silica with 50 $\mathrm{mL}$ of $\mathrm{Et}_{2} \mathrm{O}$, the solvent removed with a rotary evaporator, and the residue placed under vacuum $(<0.1$ torr) overnight. The product was purified by flash chromatography, eluting with the indicated solvent system to afford the pure tetrahydrofuran.

General procedure (B) for the Lewis acid catalyzed cycloaddition. In an inert atmosphere glovebox, a flame-dried vial was charged with $0.017 \mathrm{mmol}$ of $\mathrm{Sn}(\mathrm{OTf})_{2}$ ( 0.050 equiv) and a magnetic stir bar. Outside of the glove box, the vial was placed under an argon atmosphere, charged with $0.5 \mathrm{~mL}$ of $\mathrm{CH}_{2} \mathrm{Cl}_{2}$ and cooled to $-10{ }^{\circ} \mathrm{C}$ with stirring. After $5 \mathrm{~min}$, the stirring suspension was charged with $0.34 \mathrm{mmol}$ of the cyclopropane (1.0 equiv) via syringe, followed by $1.0 \mathrm{mmol}$ of the aldehyde (3.0 equiv). The reaction was stirred at $-10{ }^{\circ} \mathrm{C}$ until the disappearance of starting material was confirmed by TLC. The reaction mixture was then passed over a plug of silica with 50 $\mathrm{mL}$ of $\mathrm{Et}_{2} \mathrm{O}$, the solvent removed with a rotary evaporator, and the residue placed under vacuum $(<0.1$ torr) overnight. The product was purified by flash chromatography, eluting with the indicated solvent system to afford the pure tetrahydrofuran.

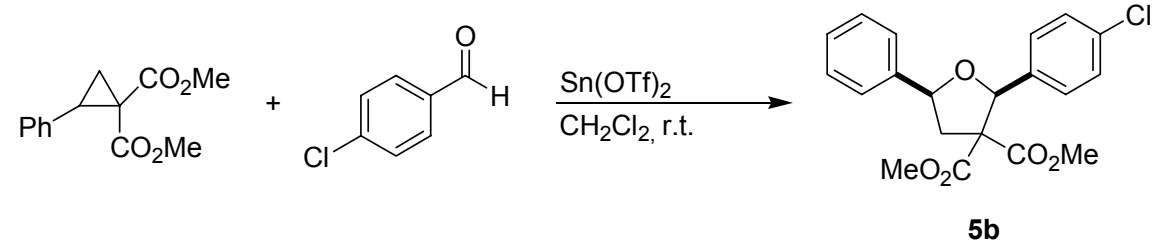

2-(4-chlorophenyl)-5-phenyltetrahydrofuran-3,3-dicarboxylic acid dimethyl ester (5b, Table 1, entry 2). In an inert atmosphere glovebox, a flame-dried vial was charged with $\mathrm{Sn}(\mathrm{OTf})_{2}(7.1 \mathrm{mg}, 0.017 \mathrm{mmol}, 0.050$ equiv), the cyclopropane ( $80.0 \mathrm{mg}, 0.34$ mmol, 1.0 equiv), and a stir bar. Outside of the glovebox, the vial was placed under argon and charged with the aldehyde (144 mg, $1.0 \mathrm{mmol}, 3.0$ equiv) in $0.5 \mathrm{~mL}$ of $\mathrm{CH}_{2} \mathrm{Cl}_{2}$. The reaction was stirred at room temperature. After $4.75 \mathrm{~h}$ the reaction was passed over a small plug of silica with $50 \mathrm{~mL}$ of $\mathrm{Et}_{2} \mathrm{O}$, and the solvent was removed by rotary evaporation. ${ }^{1} \mathrm{H}$ NMR analysis of the unpurified product $(\delta 5.73 \mathrm{vs} . \delta 3.70)$ gave the diastereomeric ratio: $>69: 1$. The crude product was purified by flash chromatography with a $2.5 \%$ to $5 \%$ acetone/petroleum ether gradient to afford $125 \mathrm{mg}(97 \%)$ of the product as a slightly yellow oil.

Analytical data for 5b: IR (thin film, $\mathrm{cm}^{-1}$ ) 3034, 2953, 2883, 1732, 1599, 1491, $1435,1385,1348,1271,1232,1209,1198,1176,1089,1057,1028,1015,941,906,843$, 800, 760, 700; ${ }^{1} \mathbf{H}$ NMR $\left(400 \mathrm{MHz}, \mathrm{CDCl}_{3}\right) \delta 7.53(\mathrm{~d}, J=7.2 \mathrm{~Hz}, 2 \mathrm{H}), 7.45(\mathrm{~d}, J=8.4$ $\mathrm{Hz}, 2 \mathrm{H}), 7.39$ (dd, $J=7.1,7.1 \mathrm{~Hz}, 2 \mathrm{H}), 7.36-7.25$ (m, 3H), 5.74 (s, 1H), 4.94 (dd, $J=$ 10.4, $6.0 \mathrm{~Hz}, 1 \mathrm{H}), 3.81$ (s, 3H), 3.16 (s, 3H), 2.97 (dd, $J=13.6,10.8 \mathrm{~Hz}, 1 \mathrm{H}), 2.74$ (dd, $J$ $=13.6,6.0 \mathrm{~Hz}, 1 \mathrm{H}) ;{ }^{13} \mathbf{C} \mathbf{~ N M R}\left(100 \mathrm{MHz}, \mathrm{CDCl}_{3}\right) \delta 171.3,169.2,139.8,136.3,134.0$, $128.6,128.6,128.3,128.1,126.6,83.9,80.1,66.3,53.1,52.4,42.8$; TLC $(80 \%$ $\mathrm{CH}_{2} \mathrm{Cl}_{2}$ /petroleum ether) $\mathrm{R}_{\mathrm{f}}$ 0.56; Anal. Calcd. for $\mathrm{C}_{20} \mathrm{H}_{19} \mathrm{ClO}_{5}$ : C, 64.09; $\mathrm{H}, 5.11$. Found: C, 64.20; H, 5.16. 


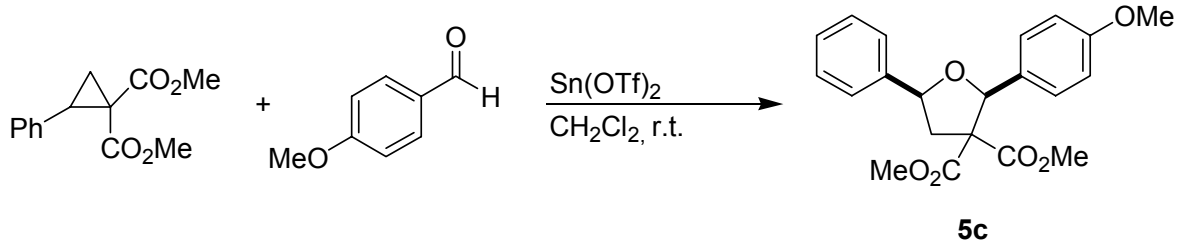

2-(4-methoxyphenyl)-5-phenyltetrahydrofuran-3,3-dicarboxylic acid dimethyl ester (5c, Table 1, entry 3). The title compound was prepared according to General Procedure A using $7.1 \mathrm{mg}$ of $\mathrm{Sn}(\mathrm{OTf})_{2}, 80.0 \mathrm{mg}$ of the cyclopropane, and $140 \mathrm{mg}$ of the aldehyde. After $3.75 \mathrm{~h}$ at room temperature and workup, ${ }^{1} \mathrm{H}$ NMR analysis of the unpurified product ( $\delta 5.75$ vs. $\delta 3.65$ ) gave the diastereomeric ratio: $>81: 1$. The crude product was purified by flash chromatography with a $2.5 \%$ to $5 \%$ to $10 \%$ acetone/petroleum ether gradient to afford $127 \mathrm{mg}(100 \%)$ of the product as a slightly yellow oil.

Analytical data for 5c: IR (thin film, $\mathrm{cm}^{-1}$ ) 3003, 2953, 2839, 1732, 1614, 1585, $1514,1435,1387,1358,1273,1250,1211,1174,1092,1057,1032,939,904,841,804$, 760,$700 ;{ }^{1} \mathbf{H}$ NMR $\left(400 \mathrm{MHz}, \mathrm{CDCl}_{3}\right) \delta 7.54(\mathrm{~d}, J=7.2 \mathrm{~Hz}, 2 \mathrm{H}), 7.48-7.29(\mathrm{~m}, 5 \mathrm{H})$, $6.84(\mathrm{~d}, J=8.8 \mathrm{~Hz}, 2 \mathrm{H}), 5.74(\mathrm{~s}, 1 \mathrm{H}), 4.93(\mathrm{dd}, J=10.4,6.0 \mathrm{~Hz}, 1 \mathrm{H}), 3.80(\mathrm{~s}, 3 \mathrm{H}), 3.76$ (s, 3H), $3.15(\mathrm{~s}, 3 \mathrm{H}), 2.98(\mathrm{dd}, J=13.2,10.8 \mathrm{~Hz}, 1 \mathrm{H}), 2.72(\mathrm{dd}, J=13.6,6.0 \mathrm{~Hz}, 1 \mathrm{H})$; ${ }^{13}$ C NMR $\left(100 \mathrm{MHz}, \mathrm{CDCl}_{3}\right) \delta 171.5,169.4,159.6,140.1,129.8,128.6,128.4,128.1$, $126.5,113.3,84.4,79.8,66.3,55.3,52.9,52.3,42.8$; TLC (20\% EtOAc/petroleum ether) $\mathrm{R}_{\mathrm{f}}$ 0.37; Anal. Calcd. for $\mathrm{C}_{21} \mathrm{H}_{22} \mathrm{O}_{6}: \mathrm{C}, 68.10 ; \mathrm{H}, 5.99$. Found: C, 68.07; H, 5.94.

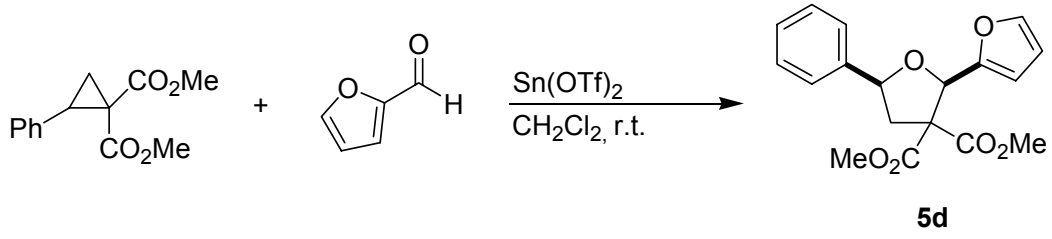

5-phenyl-1,2,3,4-tetrahydro[2,2']byfuryl-3,3-dicarboxylic acid dimethyl ester (5d, Table 1, entry 4). The title compound was prepared according to General Procedure A using $7.1 \mathrm{mg}$ of $\mathrm{Sn}(\mathrm{OTf})_{2}, 80.0 \mathrm{mg}$ of the cyclopropane, and $99 \mathrm{mg}$ of the aldehyde. After $2.75 \mathrm{~h}$ at room temperature and workup, ${ }^{1} \mathrm{H}$ NMR analysis of the unpurified product ( $\delta 4.93$ vs. $\delta 3.67$ ) gave the diastereomeric ratio: $25.9: 1$. The crude product was purified by flash chromatography with 5\% acetone/petroleum ether to afford $94 \mathrm{mg}$ $(83 \%)$ of the product as a colorless oil.

Analytical data for 5d: IR (thin film, $\mathrm{cm}^{-1}$ ) 2954, 2359, 2343, 1736, 1497, 1450, $1435,1333,1273,1232,1209,1173,1151,1093,1051,1011,945,895,808,746,700$, 669; ${ }^{1} \mathbf{H}$ NMR $\left(400 \mathrm{MHz}, \mathrm{CDCl}_{3}\right) \delta 7.47(\mathrm{~d}, J=7.2 \mathrm{~Hz}, 2 \mathrm{H}), 7.43-7.27(\mathrm{~m}, 4 \mathrm{H}), 6.38(\mathrm{~d}$, $J=3.1 \mathrm{~Hz}, 1 \mathrm{H}), 6.35-6.29(\mathrm{~m}, 1 \mathrm{H}), 5.85(\mathrm{~s}, 1 \mathrm{H}), 4.93(\mathrm{dd}, J=11.2,5.6 \mathrm{~Hz}, 1 \mathrm{H}), 3.83(\mathrm{~s}$, $3 \mathrm{H}), 3.44(\mathrm{~s}, 3 \mathrm{H}), 3.04(\mathrm{dd}, J=13.2,11.6 \mathrm{~Hz}, 1 \mathrm{H}), 2.72(\mathrm{dd}, J=13.6,5.6 \mathrm{~Hz}, 1 \mathrm{H}) ;{ }^{13} \mathbf{C}$ NMR $\left(100 \mathrm{MHz}, \mathrm{CDCl}_{3}\right) \delta 170.7,168.3,151.5,142.8,139.9,128.5,128.1,126.5,110.4$, 109.1, 80.2, 78.1, 65.2, 53.3, 52.8, 41.9; TLC (5\% acetone/petroleum ether) $\mathrm{R}_{\mathrm{f}} 0.11$; Anal. Calcd. for $\mathrm{C}_{18} \mathrm{H}_{18} \mathrm{O}_{6}$ : C, 65.45; H, 5.49. Found: C, 65.73; H, 5.56. 


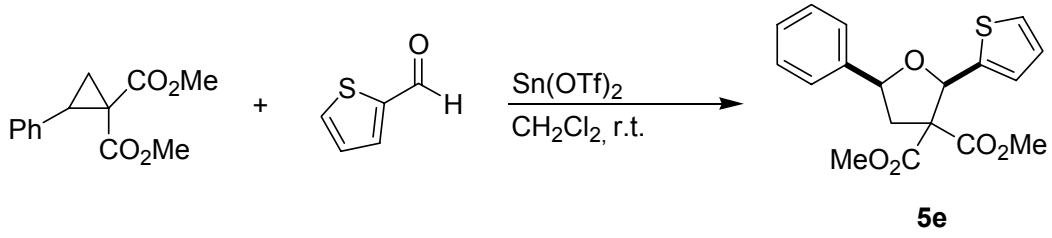

5-phenyl-2-(2-thienyl)tetrahydrofuran-3,3-dicarboxylic acid dimethyl ester (5e, Table 1, entry 5). The title compound was prepared according to General Procedure A using $7.1 \mathrm{mg}$ of $\mathrm{Sn}(\mathrm{OTf})_{2}, 80.0 \mathrm{mg}$ of the cyclopropane, and $115 \mathrm{mg}$ of the aldehyde. After $3 \mathrm{~h}$ at room temperature and workup, ${ }^{1} \mathrm{H}$ NMR of the unpurified product $(\delta 6.07 \mathrm{vs}$. $\delta 3.68)$ gave the diastereomeric ratio: $>76: 1$. The crude product was purified by flash chromatography with $7.5 \%$ acetone/petroleum ether to afford $116 \mathrm{mg}(98 \%)$ of the product as a colorless oil.

Analytical data for 5e: IR (thin film, $\mathrm{cm}^{-1}$ ) 3068, 3034, 3007, 2953, 2359, 1732, 1498, 1439, 1367, 1321, 1273, 1236, 1213, 1198, 1174, 1092, 1072, 1051, 1024, 943, $899,858,841,825,806,758,700 ;{ }^{1} \mathbf{H}$ NMR $\left(400 \mathrm{MHz}, \mathrm{CDCl}_{3}\right) \delta 7.52(\mathrm{~d}, J=7.2 \mathrm{~Hz}$, $2 \mathrm{H}), 7.38(\mathrm{dd}, J=7.2,7.2 \mathrm{~Hz}, 2 \mathrm{H}), 7.34-7.28(\mathrm{~m}, 1 \mathrm{H}), 7.24(\mathrm{~d}, J=4.8 \mathrm{~Hz}, 1 \mathrm{H}), 7.10(\mathrm{~d}, J$ $=3.2 \mathrm{~Hz}, 1 \mathrm{H}), 6.95(\mathrm{dd}, J=4.6,3.7 \mathrm{~Hz}, 1 \mathrm{H}), 6.06(\mathrm{~s}, 1 \mathrm{H}), 4.94(\mathrm{dd}, J=10.8,6.0 \mathrm{~Hz}$, $1 \mathrm{H}), 3.83(\mathrm{~s}, 3 \mathrm{H}), 3.32(\mathrm{~s}, 3 \mathrm{H}), 2.96(\mathrm{dd}, J=13.6,10.8 \mathrm{~Hz}, 1 \mathrm{H}), 2.74(\mathrm{dd}, J=13.6,6.0$ $\mathrm{Hz}, 1 \mathrm{H}) ;{ }^{13} \mathbf{C}$ NMR $\left(100 \mathrm{MHz}, \mathrm{CDCl}_{3}\right) \delta 171.0,168.8,141.0,139.7,128.5,128.2,126.5$, 126.4, 125.7, 125.3, 81.1, 80.1, 66.5, 53.2, 52.6, 42.1; TLC $\left(80 \% \mathrm{CH}_{2} \mathrm{Cl}_{2} /\right.$ petroleum ether) $\mathrm{R}_{\mathrm{f}} 0.37$; Anal. Calcd. for $\mathrm{C}_{18} \mathrm{H}_{18} \mathrm{O}_{5} \mathrm{~S}: \mathrm{C}, 62.41 ; \mathrm{H}, 5.24$. Found: $\mathrm{C}, 62.20 ; \mathrm{H}, 5.28$.

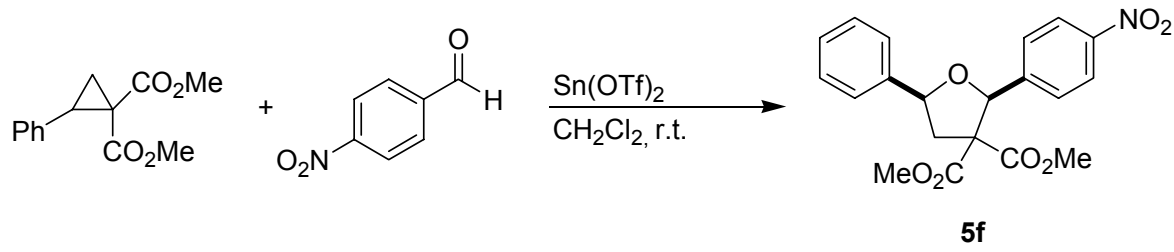

2-(4-nitrophenyl)-5-phenyltetrahydrofuran-3,3-dicarboxylic acid dimethyl ester (5f, Table 1, entry 6). In an inert atmosphere glovebox, a flame-dried vial was charged with $\mathrm{Sn}(\mathrm{OTf})_{2}(28.5 \mathrm{mg}, 0.0684 \mathrm{mmol}, 0.200$ equiv), the cyclopropane $(80.0 \mathrm{mg}, 0.34 \mathrm{mmol}$, 1.0 equiv), and a stir bar. Outside of the glovebox, the vial was placed under argon and charged with the aldehyde (155 mg, $1.0 \mathrm{mmol}, 3.0$ equiv) in $1.0 \mathrm{~mL}$ of $\mathrm{CH}_{2} \mathrm{Cl}_{2}$. The reaction was stirred at room temperature. After $15 \mathrm{~h}$ the reaction was passed over a small plug of silica with $50 \mathrm{~mL}$ of $\mathrm{Et}_{2} \mathrm{O}$, and the solvent was removed by rotary evaporation. ${ }^{1} \mathrm{H}$ NMR analysis of the unpurified product ( $\delta 5.83$ vs. $\delta 3.73$ ) gave the diastereomeric ratio: $>15: 1$. The crude product was purified by flash chromatography with a $40 \%$ to $50 \%$ to $60 \% \mathrm{CH}_{2} \mathrm{Cl}_{2} /$ petroleum ether gradient to afford $120 \mathrm{mg}(91 \%)$ of the product as a white solid.

Analytical data for 5f: $\mathrm{mp} 112-115{ }^{\circ} \mathrm{C}$; IR (thin film, $\mathrm{cm}^{-1}$ ) 2954, 1732, 1606, 1524, 1495, 1435, 1387, 1348, 1313, 1273, 1232, 1209, 1176, 1109, 1092, 1059, 1030, $1016,941,862,845,806,762,746,698 ;{ }^{1} \mathbf{H}$ NMR $\left(400 \mathrm{MHz}, \mathrm{CDCl}_{3}\right) \delta 8.18(\mathrm{~d}, J=8.8$ $\mathrm{Hz}, 2 \mathrm{H}), 7.72(\mathrm{~d}, J=8.4 \mathrm{~Hz}, 2 \mathrm{H}), 7.55(\mathrm{~d}, J=7.2 \mathrm{~Hz}, 2 \mathrm{H}), 7.42(\mathrm{dd}, J=7.2,7.2 \mathrm{~Hz}, 2 \mathrm{H})$, 
7.39-7.31 (m, 1H), $5.82(\mathrm{~s}, 1 \mathrm{H}), 4.99(\mathrm{dd}, J=10.0,6.8 \mathrm{~Hz}, 1 \mathrm{H}), 3.85(\mathrm{~s}, 3 \mathrm{H}), 3.15(\mathrm{~s}$, $3 \mathrm{H}), 3.00(\mathrm{dd}, J=13.2,10.8 \mathrm{~Hz}, 1 \mathrm{H}), 2.81(\mathrm{dd}, J=13.2,6.4 \mathrm{~Hz}, 1 \mathrm{H}) ;{ }^{13} \mathbf{C}$ NMR $(100$ $\left.\mathrm{MHz}, \mathrm{CDCl}_{3}\right) \delta 171.0,169.0,147.8,145.0,139.3,128.7,128.5,128.1,126.6,123.0$, 83.5, 80.4, 66.4, 53.3, 52.5, 42.9; TLC (20\% EtOAc/petroleum ether) $\mathrm{R}_{\mathrm{f}} 0.36$; Anal. Calcd. for $\mathrm{C}_{20} \mathrm{H}_{19} \mathrm{NO}_{7}$ : C, 62.33; H, 4.97; N, 3.63. Found: C, 62.05; H, 4.96; N, 3.65.

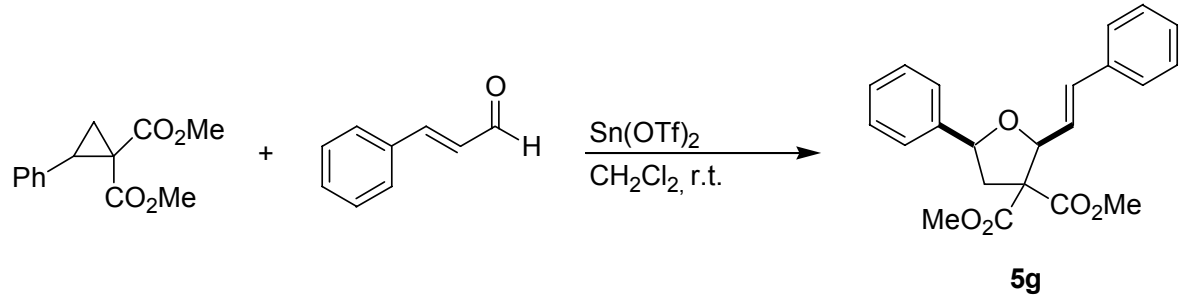

5-phenyl-2-styryltetrahydrofuran-3,3-dicarboxylic acid dimethyl ester (5g, Table 1, entry 7). The title compound was prepared according to General Procedure A using 7.1 $\mathrm{mg}$ of $\mathrm{Sn}(\mathrm{OTf})_{2}, 80.0 \mathrm{mg}$ of the cyclopropane, and $137 \mathrm{mg}$ of the aldehyde. After $2.5 \mathrm{~h}$ at room temperature and workup, ${ }^{1} \mathrm{H}$ NMR of the unpurified product ( $\delta 4.91$ vs. $\delta 3.69$ ) gave the diastereomeric ratio: 17.8:1. The isomers were separated and purified by flash chromatography with 5\% acetone/petroleum ether to afford $119 \mathrm{mg}$ (95\%) of the products as yellow oils.

Analytical data for 5g: IR (thin film, cm ${ }^{-1}$ ) 3084, 3057, 3030, 3003, 2953, 2927, $2848,1732,1657,1601,1578,1497,1450,1435,1387,1354,1335,1273,1225,1198$, $1176,1140,1090,1086,1043,1005,970,945,914,845,818,796,760,748,696 ;{ }^{1} \mathbf{H}$ NMR $\left(500 \mathrm{MHz}, \mathrm{CDCl}_{3}\right) \delta 7.50-7.45(\mathrm{~m}, 2 \mathrm{H}), 7.40-7.20(\mathrm{~m}, 8 \mathrm{H}), 6.80(\mathrm{~d}, J=16.0 \mathrm{~Hz}$, $1 \mathrm{H}), 6.23(\mathrm{dd}, J=16.0,7.0 \mathrm{~Hz}, 1 \mathrm{H}), 5.28(\mathrm{dd}, J=7.0,1.0 \mathrm{~Hz}, 1 \mathrm{H}), 4.91(\mathrm{dd}, J=10.5$, $6.5 \mathrm{~Hz}, 1 \mathrm{H}), 3.84(\mathrm{~s}, 3 \mathrm{H}), 3.62(\mathrm{~s}, 3 \mathrm{H}), 2.85(\mathrm{dd}, J=13.5,10.0 \mathrm{~Hz}, 1 \mathrm{H}), 2.77$ (dd, $J=$ 13.5, $6.5 \mathrm{~Hz}, 1 \mathrm{H}) ;{ }^{13} \mathrm{C}$ NMR $\left(125 \mathrm{MHz}, \mathrm{CDCl}_{3}\right) \delta 171.0,169.4,140.2,136.5,133.1$, $128.7,128.6,128.2,128.0,126.8,126.6,125.0,83.2,80.2,65.3,53.2,52.9,42.3$; TLC $\left(80 \% \mathrm{CH}_{2} \mathrm{Cl}_{2}\right.$ /petroleum ether) $\mathrm{R}_{\mathrm{f}}$ 0.38; Anal. Calcd. for $\mathrm{C}_{22} \mathrm{H}_{22} \mathrm{O}_{5}: \mathrm{C}, 72.12 ; \mathrm{H}, 6.05$. Found: C, 72.25; H, 6.20.

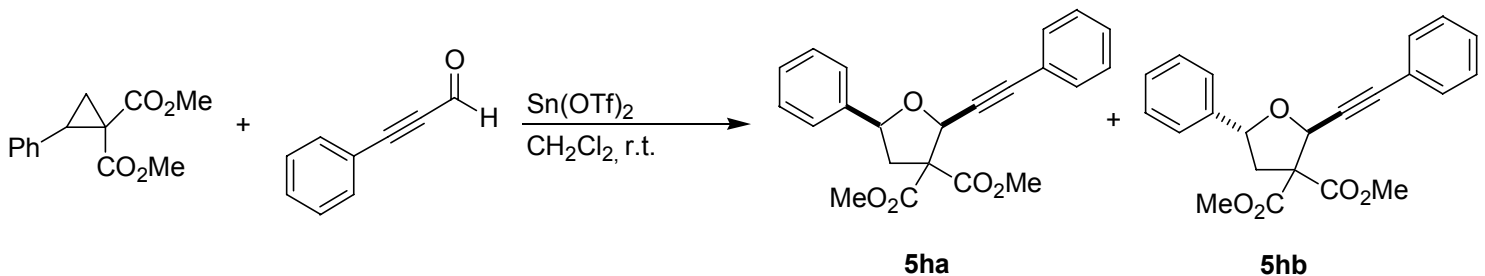

cis-5-phenyl-2-phenylethynyltetrahydrofuran-3,3-dicarboxylic acid dimethyl ester (5ha, Table 1, entry 8). In an inert atmosphere glovebox, a flame-dried vial was charged with $\mathrm{Sn}(\mathrm{OTf})_{2}(14.2 \mathrm{mg}, 0.034 \mathrm{mmol}, 0.10$ equiv), the cyclopropane $(80.0 \mathrm{mg}$, $0.34 \mathrm{mmol}, 1.0$ equiv), and a stir bar. Outside of the glovebox, the vial was placed under argon and charged with $0.5 \mathrm{~mL}$ of $\mathrm{CH}_{2} \mathrm{Cl}_{2}$ and the aldehyde $(133 \mathrm{mg}, 1.0 \mathrm{mmol}, 3.0$ equiv). The reaction was stirred at room temperature. After $5.5 \mathrm{~h}$ the reaction was passed over a small plug of silica with $50 \mathrm{~mL}$ of $\mathrm{Et}_{2} \mathrm{O}$, the solvent removed by rotary evaporation, and the residue placed under vacuum $(<0.1$ torr $)$ overnight. ${ }^{1} \mathrm{H} \mathrm{NMR}$ analysis of the unpurified product ( $\delta 5.67$ vs. $\delta 5.93)$ gave the diastereomeric ratio: 1.5:1. 
The isomers were separated and purified by flash chromatography with $7.5 \%$ acetone/petroleum ether to afford $118 \mathrm{mg}(94 \%)$ of the products as yellow oils.

Analytical data for 5ha: IR (thin film, $\mathrm{cm}^{-1}$ ) 3064, 3032, 3005, 2953, 2231, 1738, 1599, 1574, 1491, 1435, 1383, 1354, 1327, 1273, 1228, 1196, 1173, 1092, 10701049 , $1028,1001,989,955,914,887,843,804,758,692 ;{ }^{1} \mathbf{H}$ NMR $\left(400 \mathrm{MHz}, \mathrm{CDCl}_{3}\right) \delta 7.60-$ $7.23(\mathrm{~m}, 10 \mathrm{H}), 5.66(\mathrm{~s}, 1 \mathrm{H}), 4.96(\mathrm{dd}, J=10.8,6.0 \mathrm{~Hz}, 1 \mathrm{H}), 3.86(\mathrm{~s}, 3 \mathrm{H}), 3.77(\mathrm{~s}, 3 \mathrm{H})$, $2.93(\mathrm{dd}, J=13.2,10.8 \mathrm{~Hz}, 1 \mathrm{H}), 2.82(\mathrm{dd}, J=13.2,5.6 \mathrm{~Hz}, 1 \mathrm{H}) ;{ }^{13} \mathbf{C}$ NMR $(100 \mathrm{MHz}$, $\left.\mathrm{CDCl}_{3}\right) \delta 169.8,168.0,140.3,131.8,128.8,128.6,128.4,128.1,126.5,122.2,87.8,85.2$, 81.1, 73.0, 66.6, 53.5, 53.1, 41.5; TLC (10\% acetone/petroleum ether) $\mathrm{R}_{\mathrm{f}}$ 0.17; Anal. Calcd. for $\mathrm{C}_{22} \mathrm{H}_{20} \mathrm{O}_{5}$ : C, 72.51; H, 5.53. Found: C, 72.79; H, 5.48.

Analytical data for trans-5-phenyl-2-phenylethynyltetrahydrofuran-3,3dicarboxylic acid dimethyl ester (5hb, Table 1, entry 8): IR (thin film, $\mathrm{cm}^{-1}$ ) 3064, 3034, 3003, 2953, 2850, 2227, 1740, 1599, 1491, 1435, 1331, 1269, 1228, 1198, 1171, 1109, 1049, 1001, 987, 970, 939, 916, 889, 758, 692; ${ }^{\mathbf{1}} \mathbf{H}$ NMR (400 MHz, $\left.\mathrm{CDCl}_{3}\right)$ $\delta$ 7.50-7.24 (m, 10H), $5.91(\mathrm{~s}, 1 \mathrm{H}), 5.41(\mathrm{dd}, J=7.6,7.6 \mathrm{~Hz}, 1 \mathrm{H}), 3.79(\mathrm{~s}, 3 \mathrm{H}), 3.74(\mathrm{~s}$, $3 \mathrm{H}), 3.40(\mathrm{dd}, J=13.2,7.2 \mathrm{~Hz}, 1 \mathrm{H}), 2.23(\mathrm{dd}, J=13.6,8.4 \mathrm{~Hz}, 1 \mathrm{H}) ;{ }^{13} \mathbf{C}$ NMR $(100$ $\left.\mathrm{MHz}, \mathrm{CDCl}_{3}\right) \delta 170.1,168.4,140.5,132.0,128.9,128.6,128.4,128.0,126.0,122.2$, $87.8,84.6,80.4,73.3,66.6,53.3$ (two overlapping signals), 41.5; TLC (10\% acetone/petroleum ether) $\mathrm{R}_{\mathrm{f}}$ 0.24; Anal. Calcd. for $\mathrm{C}_{22} \mathrm{H}_{20} \mathrm{O}_{5}: \mathrm{C}, 72.51 ; \mathrm{H}, 5.53$. Found: C, 72.63; H, 5.49 .

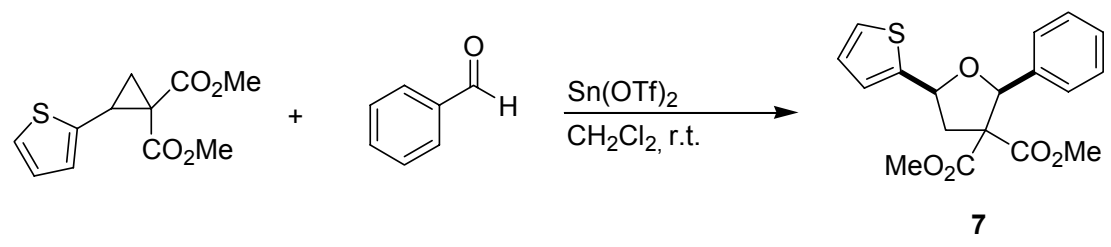

2-phenyl-5-(2-thienyl)tetrahydrofuran-3,3-dicarboxylic acid dimethyl ester (7). The title compound was prepared according to General Procedure B using $7.1 \mathrm{mg}$ of $\mathrm{Sn}(\mathrm{OTf})_{2}, 81.5 \mathrm{mg}$ of the cyclopropane, and $110 \mathrm{mg}$ of benzaldehyde. After $45 \mathrm{~min}$ at $10{ }^{\circ} \mathrm{C}$ and workup, ${ }^{1} \mathrm{H}$ NMR of the unpurified product ( $\delta 5.22 \mathrm{vs}$. $\left.\delta 2.58\right)$ gave the diastereomeric ratio: 21.7:1. The crude product was purified by flash chromatography with $10 \%$ acetone/petroleum ether to afford $113.5 \mathrm{mg}(97 \%)$ of the product as a slightly yellow oil.

Analytical data for 7: IR (thin film, $\mathrm{cm}^{-1}$ ) 2953, 1732, 1497, 1456, 1435, 1387, $1365,1327,1273,1232,1207,1174,1107,1076,1053,1028,1022,933,922,897,852$, 833, 814, 752, 700; ${ }^{1} \mathbf{H}$ NMR (400 MHz, $\left.\mathrm{CDCl}_{3}\right) \delta 7.50-7.43(\mathrm{~m}, 2 \mathrm{H}), 7.37-7.23(\mathrm{~m}, 4 \mathrm{H})$, $7.14(\mathrm{~d}, J=3.6 \mathrm{~Hz}, 1 \mathrm{H}), 7.00(\mathrm{dd}, J=5.2,3.6 \mathrm{~Hz}, 1 \mathrm{H}), 5.79(\mathrm{~s}, 1 \mathrm{H}), 5.22(\mathrm{dd}, J=10.8$, $6.0 \mathrm{~Hz}, 1 \mathrm{H}), 3.81(\mathrm{~s}, 3 \mathrm{H}), 3.15-3.09(\mathrm{~m}, 4 \mathrm{H}), 2.76(\mathrm{dd}, J=13.6,6.0 \mathrm{~Hz}, 1 \mathrm{H}) ;{ }^{13} \mathbf{C}$ NMR $\left(100 \mathrm{MHz}, \mathrm{CDCl}_{3}\right) \delta 171.2,168.9,142.7,137.6,128.3,127.9,127.1,126.7,125.7,125.5$, 84.5, 75.8, 66.4, 53.1, 52.3, 42.9; TLC $\left(80 \% \mathrm{CH}_{2} \mathrm{Cl}_{2} /\right.$ petroleum ether) $\mathrm{R}_{\mathrm{f}} 0.43$; Anal. Calcd. for $\mathrm{C}_{18} \mathrm{H}_{18} \mathrm{O}_{5} \mathrm{~S}$ : C, 62.41; H, 5.24. Found: C, 62.32; H, 5.23. 


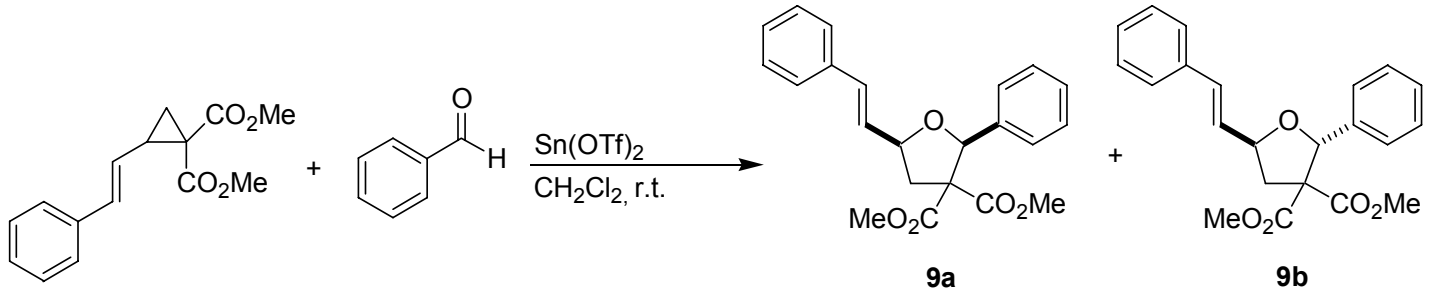

2-phenyl-5-styryltetrahydrofuran-3,3-dicarboxylic acid dimethyl ester (9a). The title compound was prepared according to General Procedure B using $7.1 \mathrm{mg}$ of $\mathrm{Sn}(\mathrm{OTf})_{2}, 89 \mathrm{mg}$ of the cyclopropane, and $110 \mathrm{mg}$ of benzaldehyde. After $1 \mathrm{~h}$ at $-10{ }^{\circ} \mathrm{C}$ and workup, ${ }^{1} \mathrm{H}$ NMR of the unpurified product ( $\delta 5.73$ vs. $\delta 5.87$ ) gave the diastereomeric ratio: $2.4: 1$. The crude product was purified by flash chromatography with a $5 \%$ to $10 \%$ acetone/petroleum ether gradient to afford $123.1 \mathrm{mg}(98 \%)$ of the product (inseparable diastereomers) as a colorless oil.

Analytical data for combined 9a/9b: IR (thin film, $\mathrm{cm}^{-1}$ ) 3082, 3062, 3001, 2953, 1732, 1601, 1578, 1497, 1452, 1435, 1360, 1335, 1269, 1228, 1207, 1174, 1109, 1090, $1070,1049,1028,968,916,881,845,825,814,752,698 ;{ }^{1} \mathbf{H}$ NMR $\left(300 \mathrm{MHz}, \mathrm{CDCl}_{3}\right)$ $\delta$ 7.54-7.18 (m, 20H, major/minor), $6.72(\mathrm{~d}, J=15.9 \mathrm{~Hz}, 1 \mathrm{H}$, major), 6.68 (d, $J=15.6$ $\mathrm{Hz}, 1 \mathrm{H}$, minor), 6.44 (dd, $J=15.9,6.9 \mathrm{~Hz}, 1 \mathrm{H}$, major), 6.25 (dd, $J=15.9,6.6 \mathrm{~Hz}, 1 \mathrm{H}$, minor), 5.87 (s, 1H, minor), 5.73 (s, 1H, major), 5.27 (ddd, $J=7.2,7.2,7.2 \mathrm{~Hz}, 1 \mathrm{H}$, minor), 4.59 (ddd, $J=9.9,6.6,6.6 \mathrm{~Hz}, 1 \mathrm{H}$, major), 3.80 (s, 3H, major), 3.74 (s, $3 \mathrm{H}$, minor), 3.17 (s, $3 \mathrm{H}$, minor), 3.11 (s, $3 \mathrm{H}$, major), 3.08 (dd, $J=13.2,6.9 \mathrm{~Hz}, 1 \mathrm{H}$, minor), 2.87 (dd, $J=13.2,10.2 \mathrm{~Hz}, 1 \mathrm{H}$, major), 2.55 (dd, $J=13.5,6.0 \mathrm{~Hz}, 1 \mathrm{H}$, major), 2.28 (dd, $J=13.2,7.5 \mathrm{~Hz}, 1 \mathrm{H}$, minor); ${ }^{13} \mathbf{C}$ NMR $\left(75 \mathrm{MHz}, \mathrm{CDCl}_{3}\right) \delta 171.2,170.4,169.2,169.1$, $138.3,137.8,136.5,136.4,133.0,131.5,129.4,128.6,128.2,128.1,128.03,127.95$, $127.89,127.86,127.6,127.0,126.8,126.62,126.55,84.3,83.6,79.9,79.2,66.29,66.26$, $53.0, \quad 52.9, \quad 52.3, \quad 52.2, \quad 41.1,40.7 \quad$ (2 overlapping $\mathrm{sp}^{2}$ signals); TLC $(80 \%$ $\mathrm{CH}_{2} \mathrm{Cl}_{2}$ /petroleum ether) $\mathrm{R}_{\mathrm{f}}$ 0.43; Anal. Calcd. for $\mathrm{C}_{22} \mathrm{H}_{22} \mathrm{O}_{5}: \mathrm{C}, 72.12 ; \mathrm{H}, 6.05$. Found: C, $72.40 ; \mathrm{H}, 6.15$.

\section{Reference}

(1) Fraser, W.; Suckling, C. J.; Wood, H. C. S. J. Chem. Soc. Perkin Trans. 1. 1990, 3137-3144. 


\section{Appendix. NMR spectra}

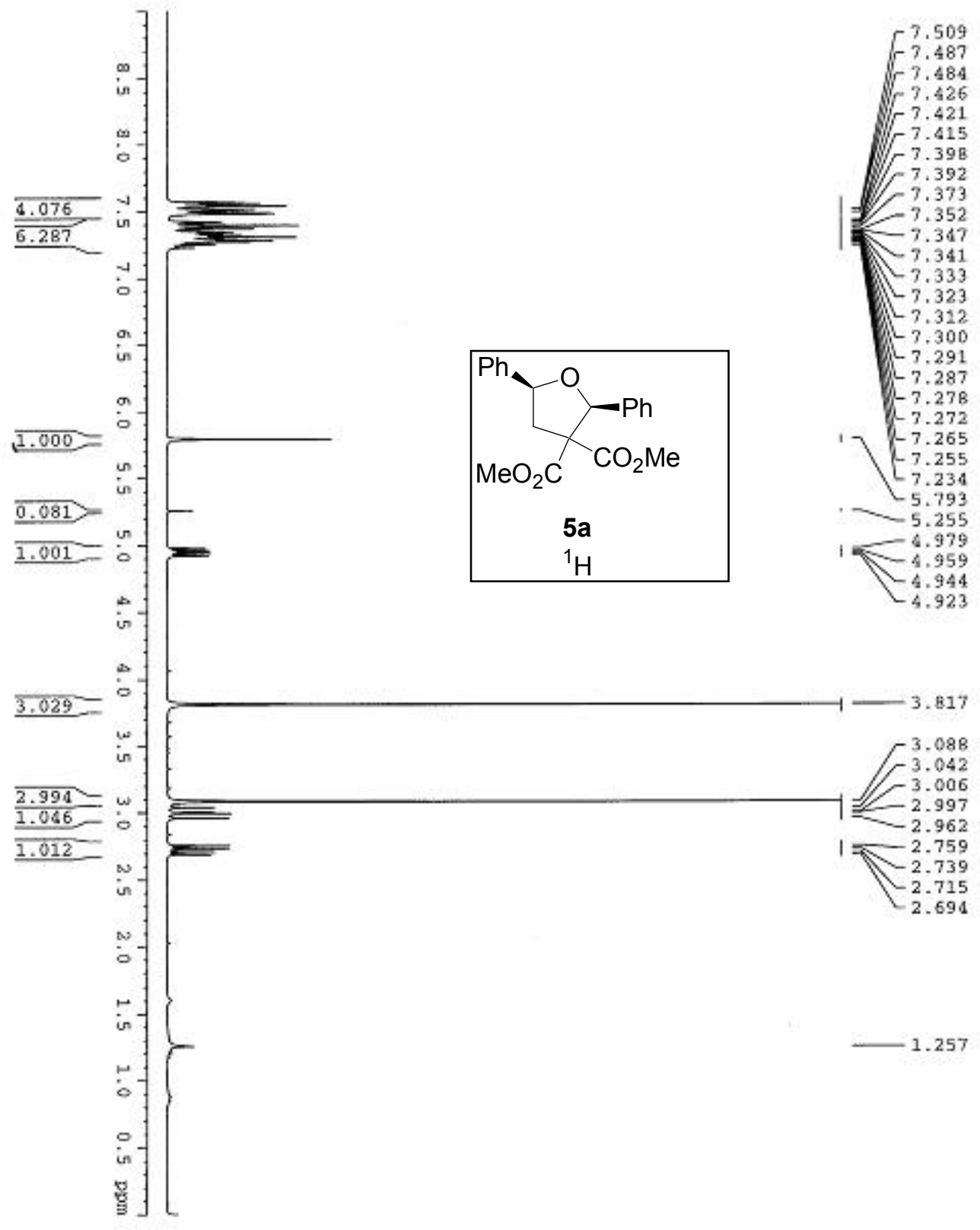




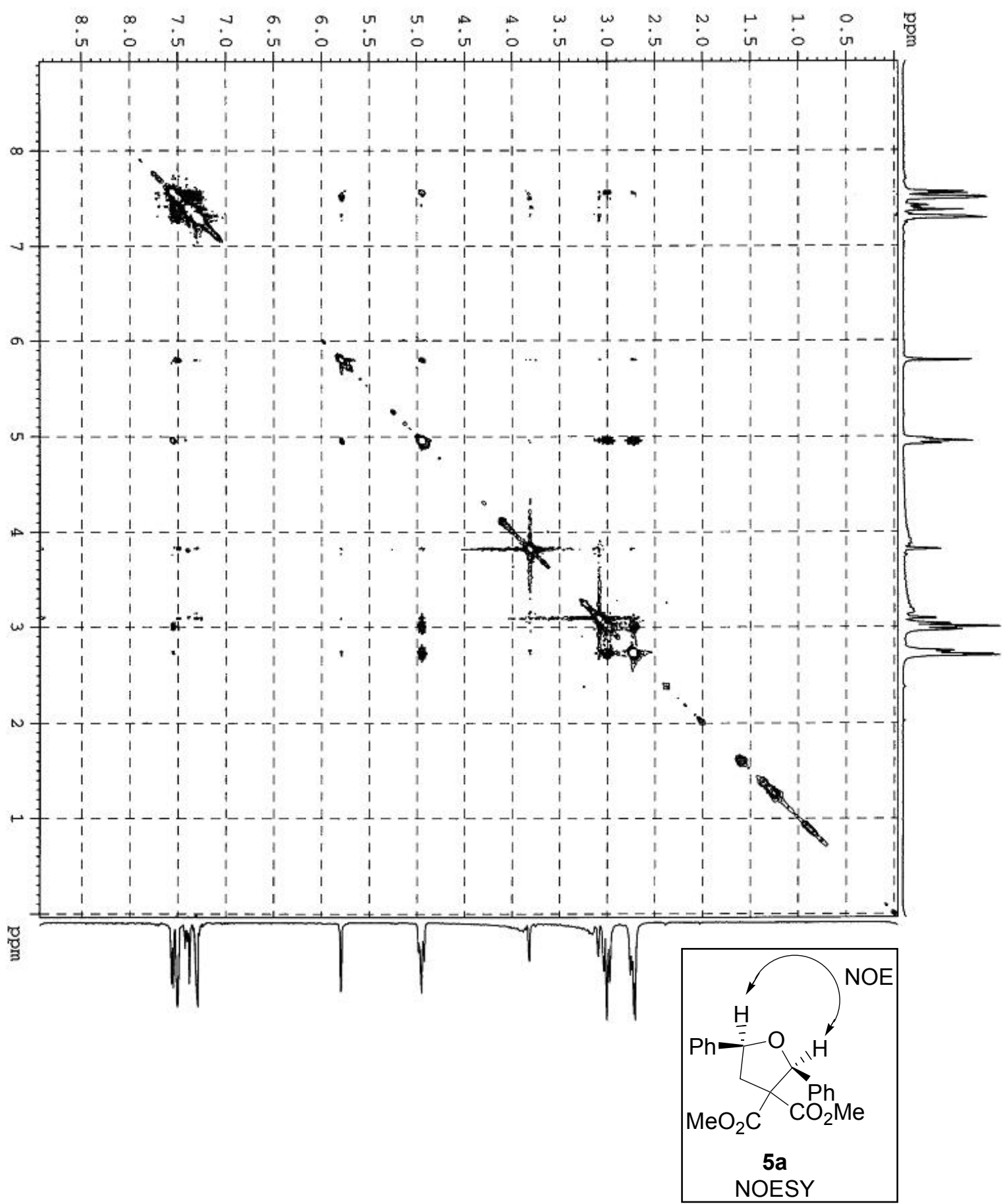




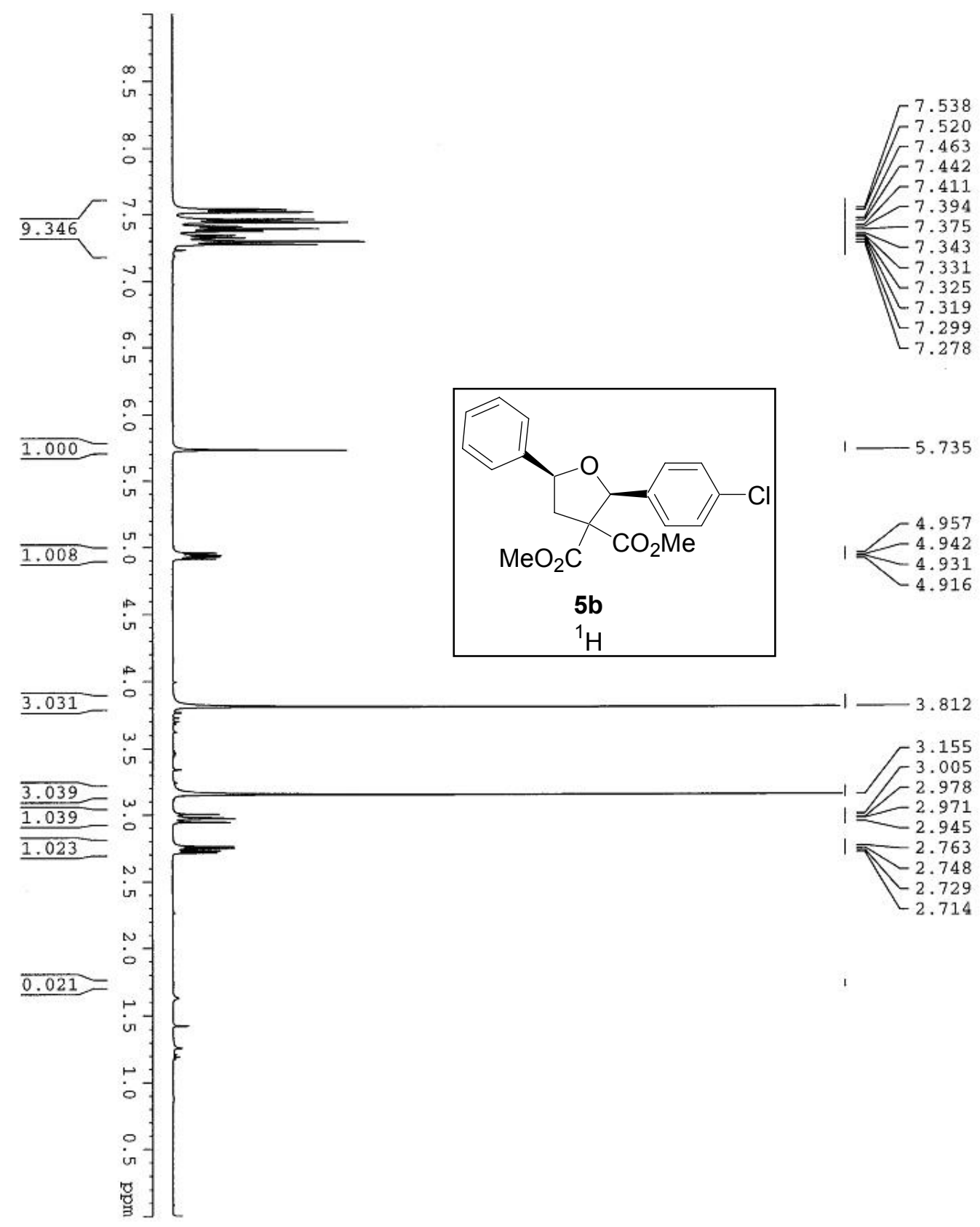




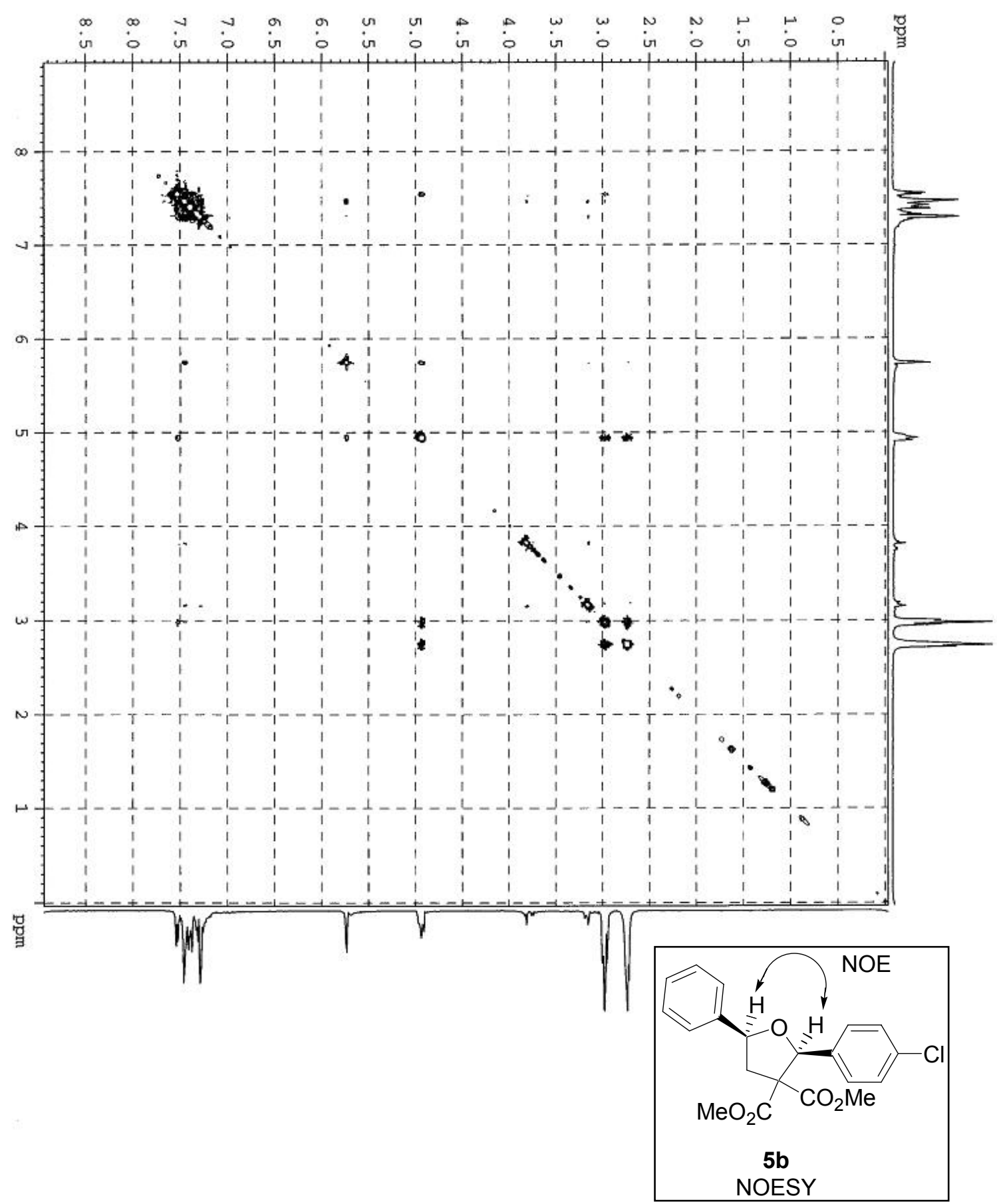




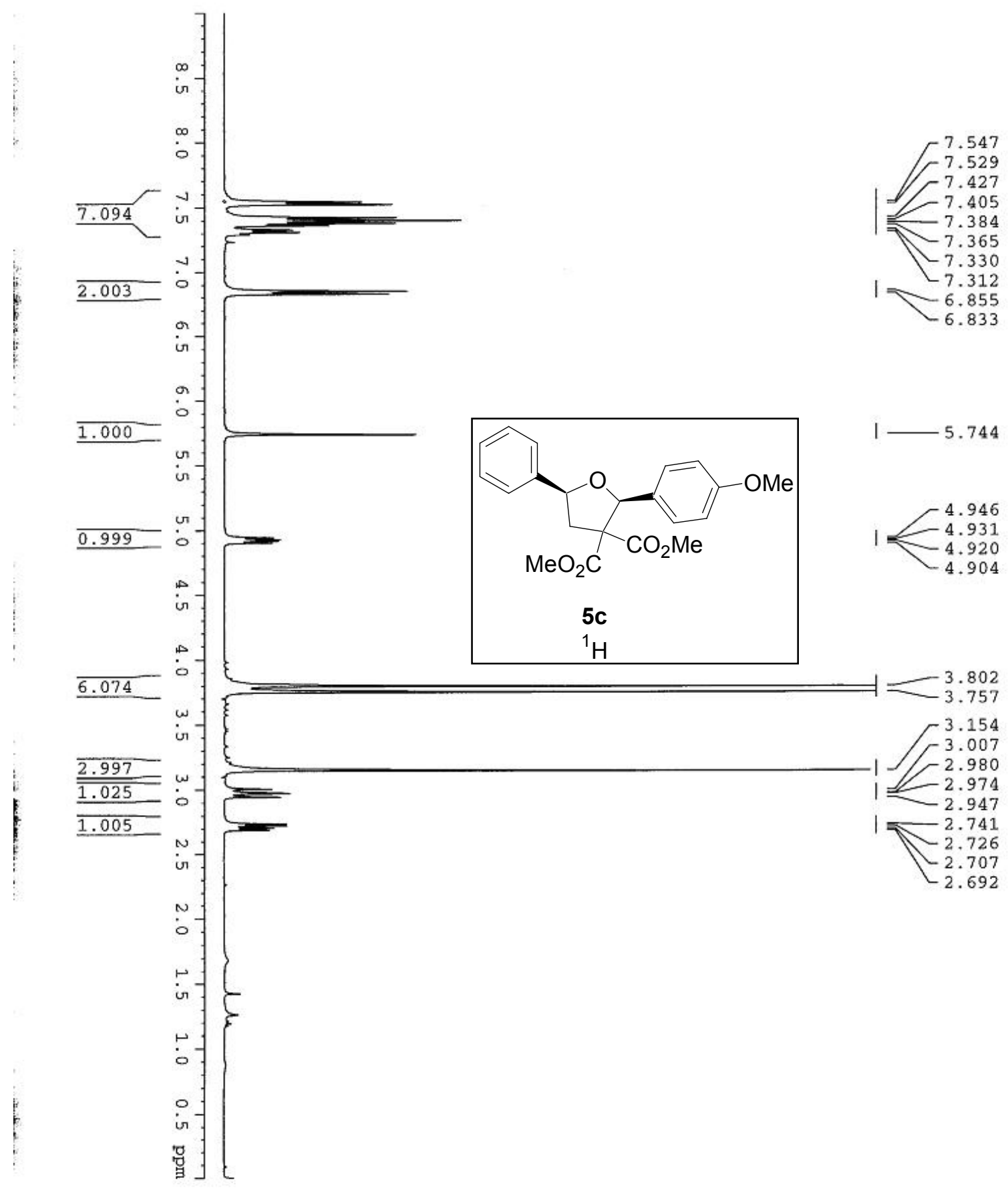




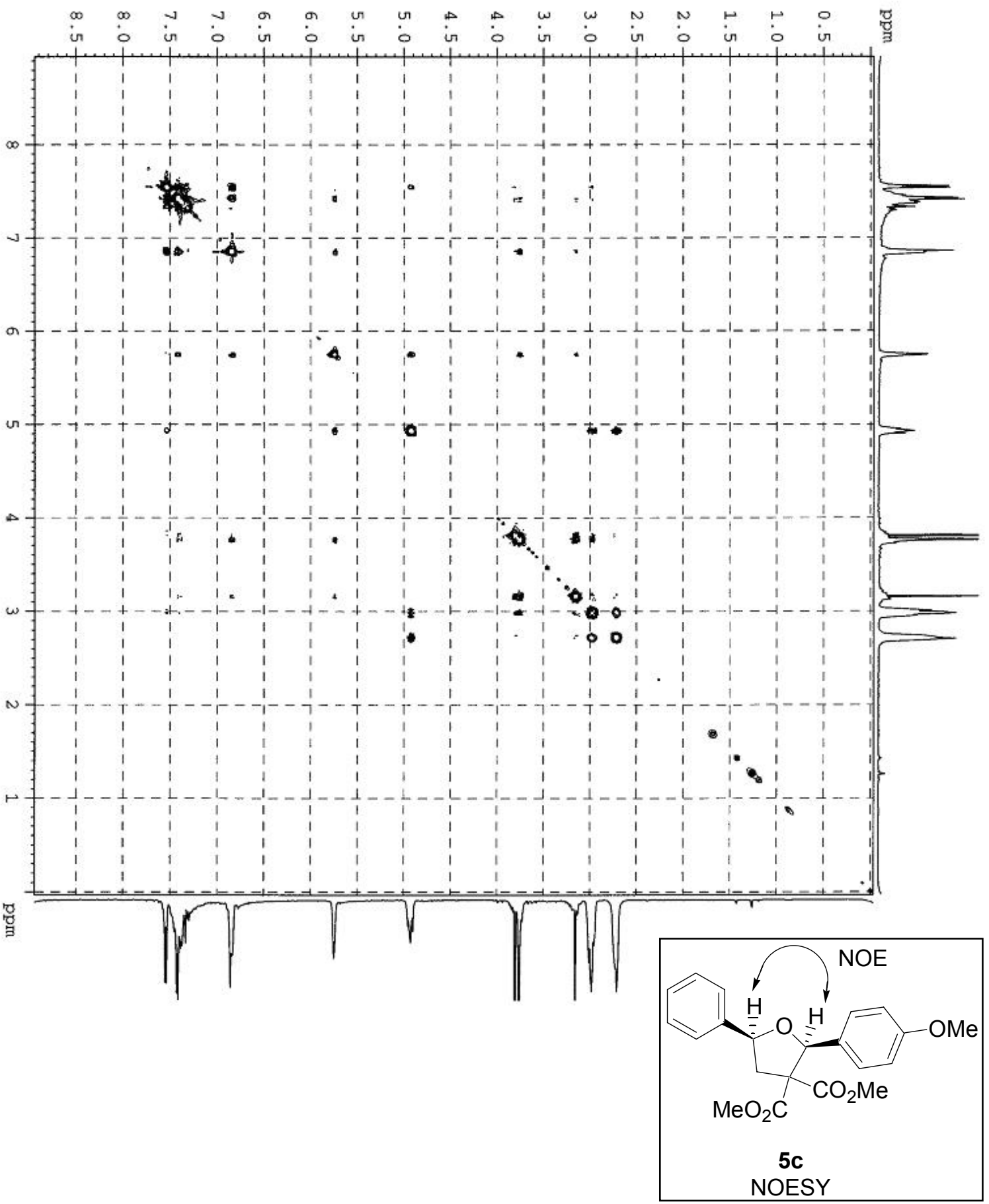




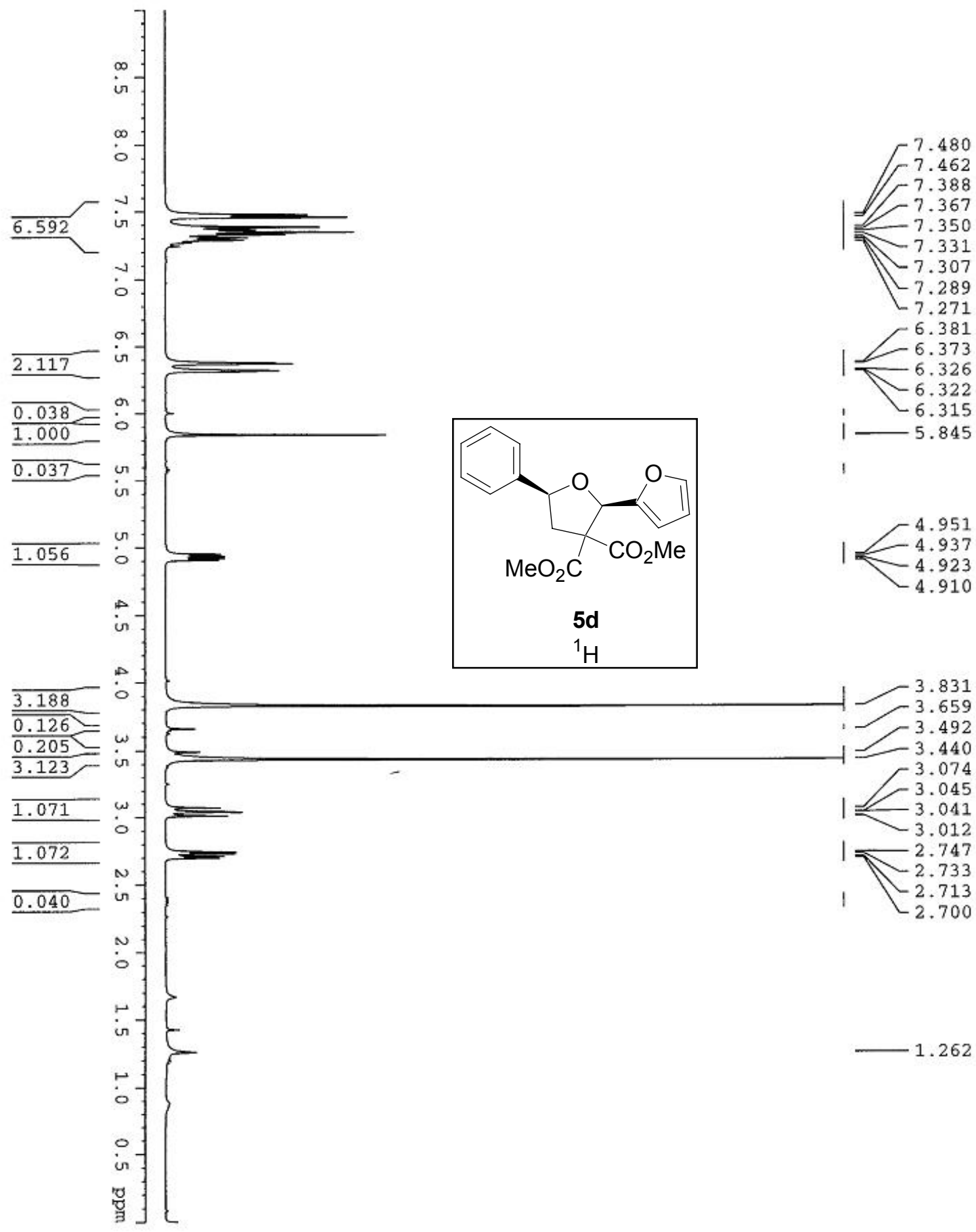




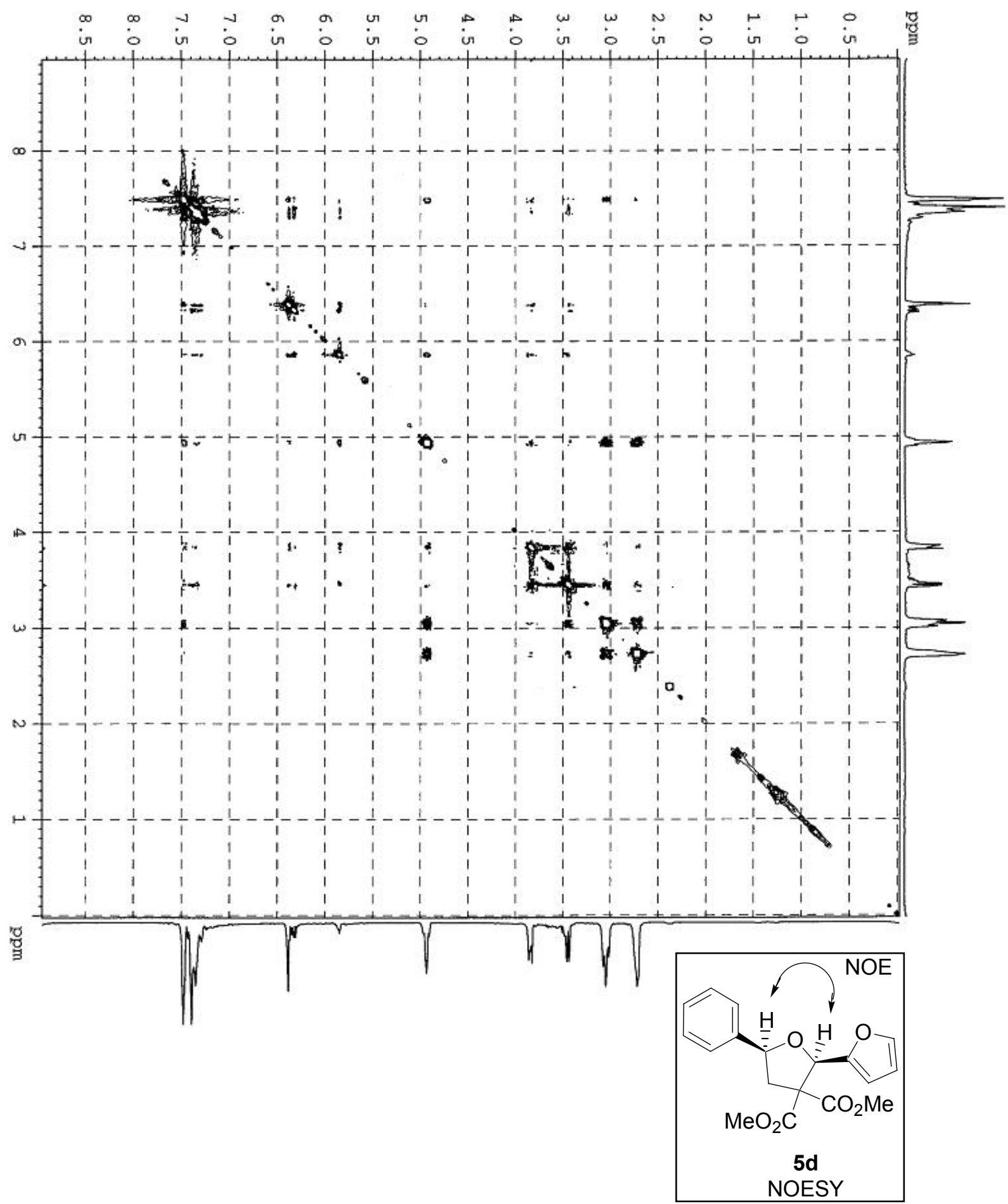




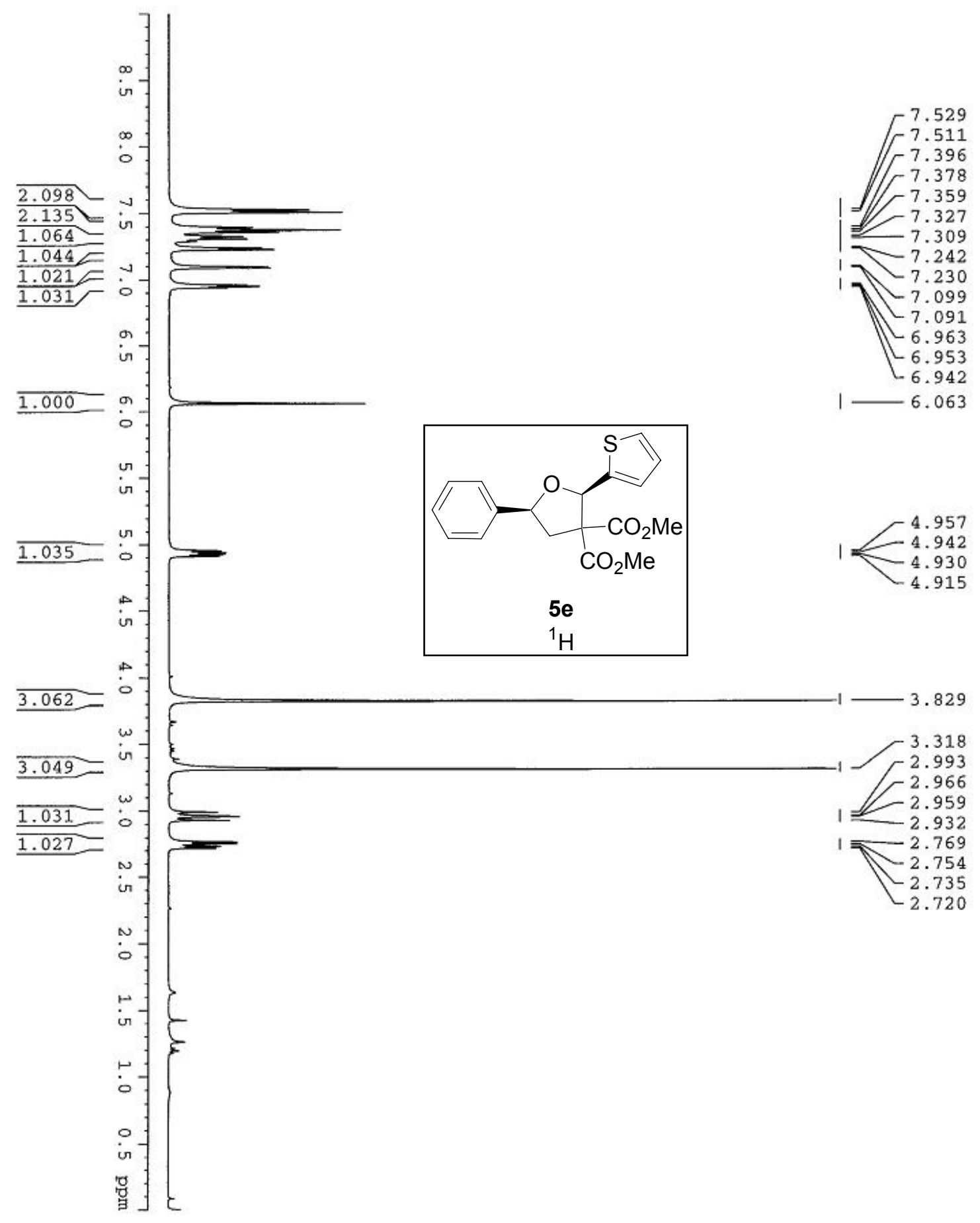



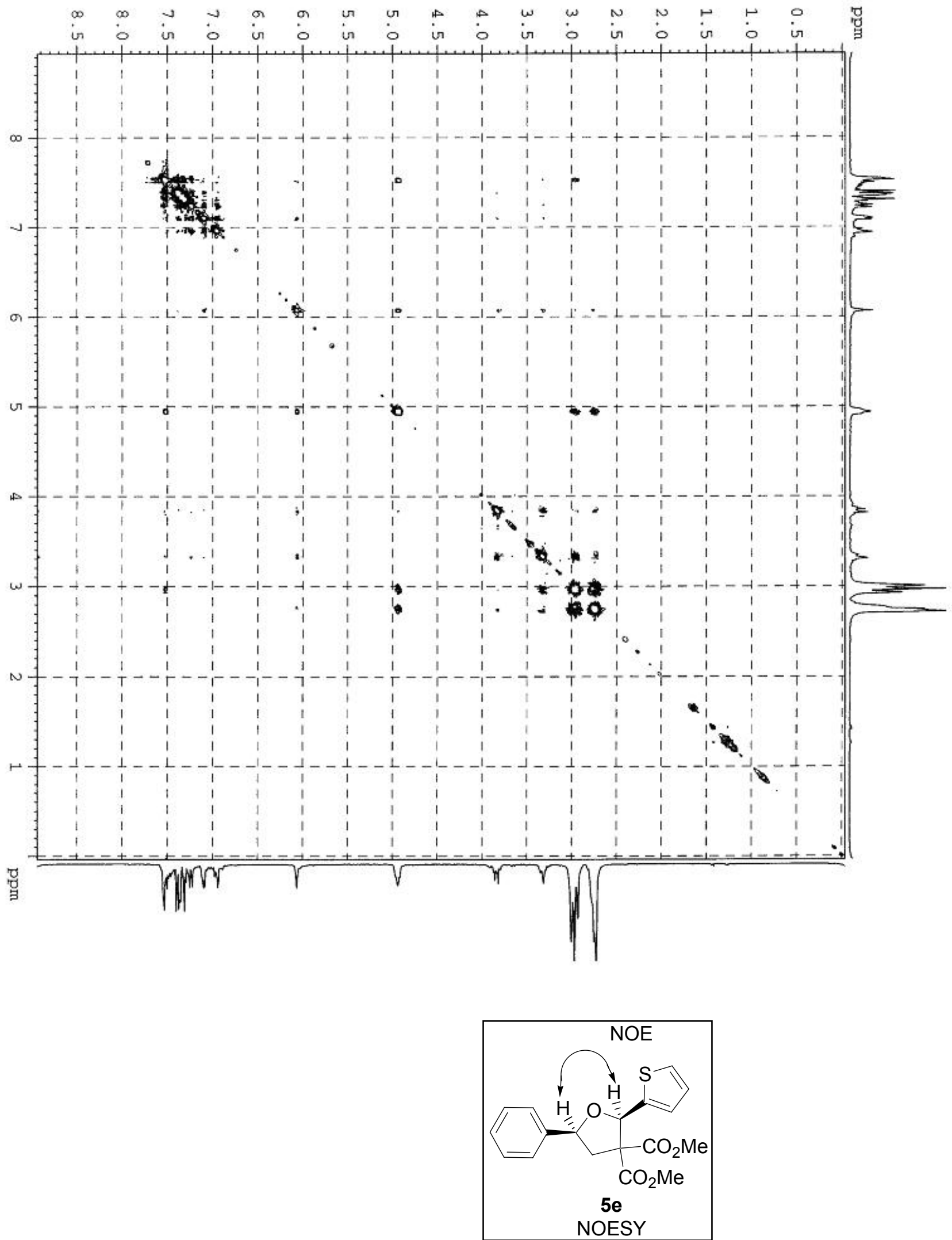


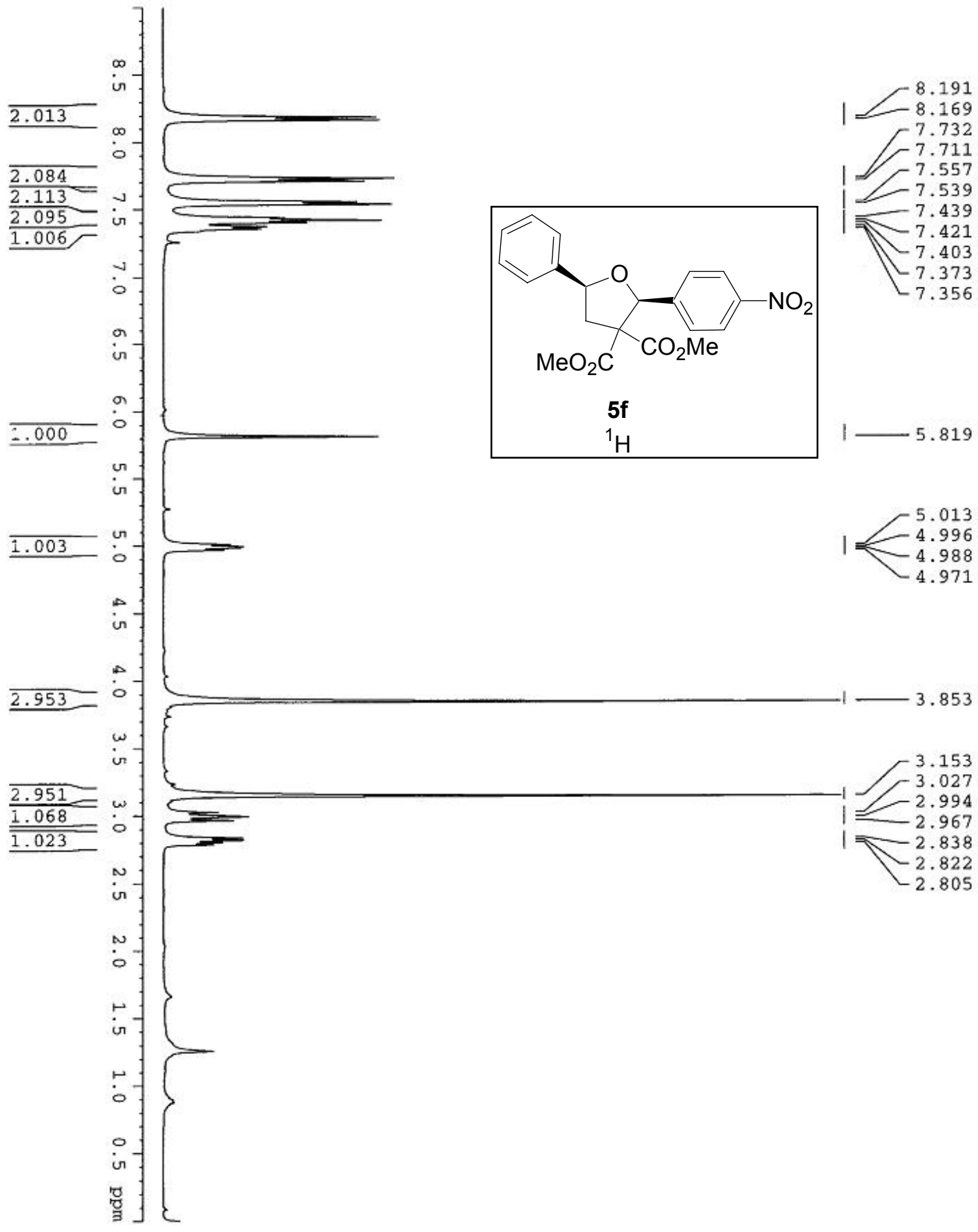




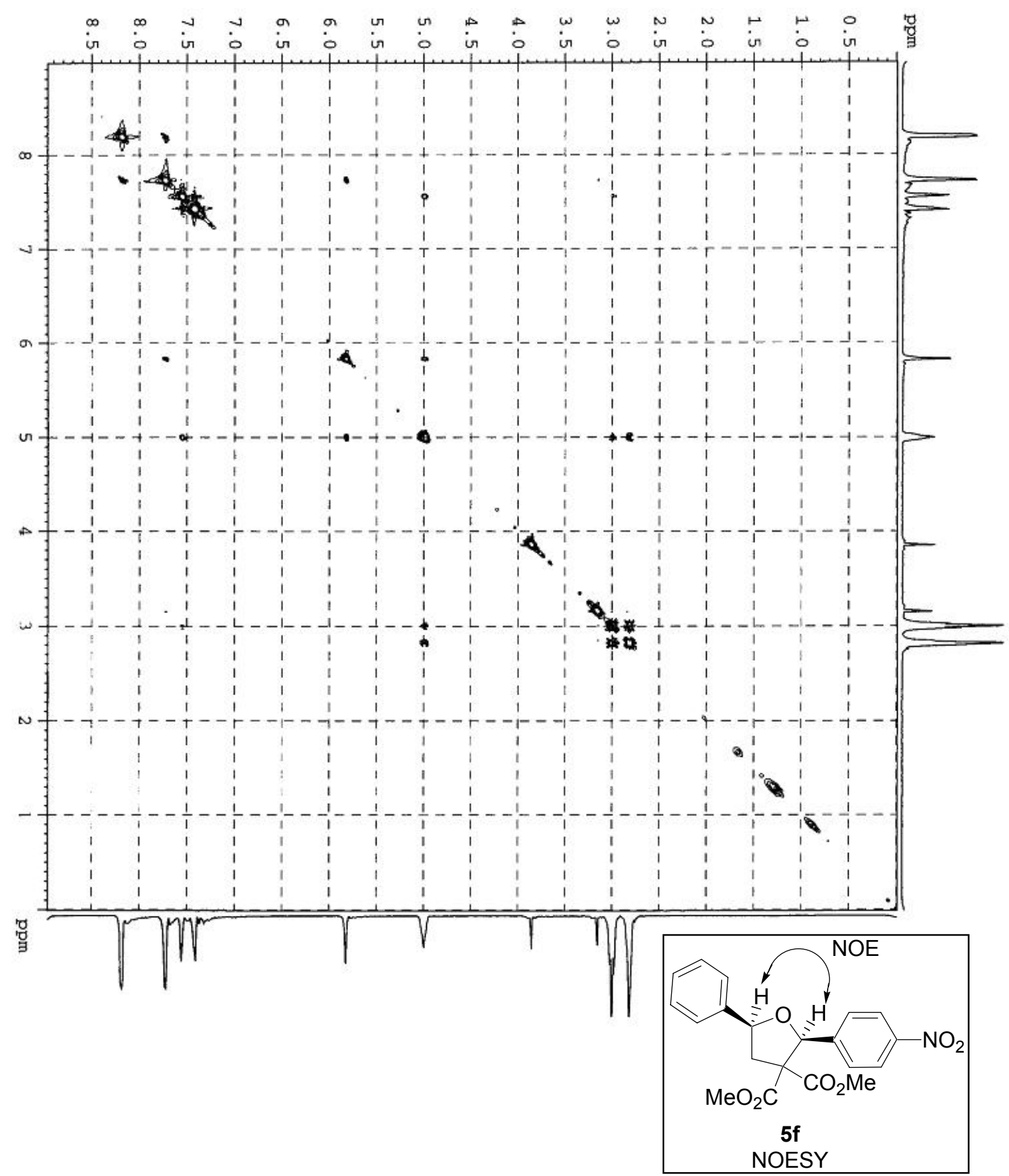




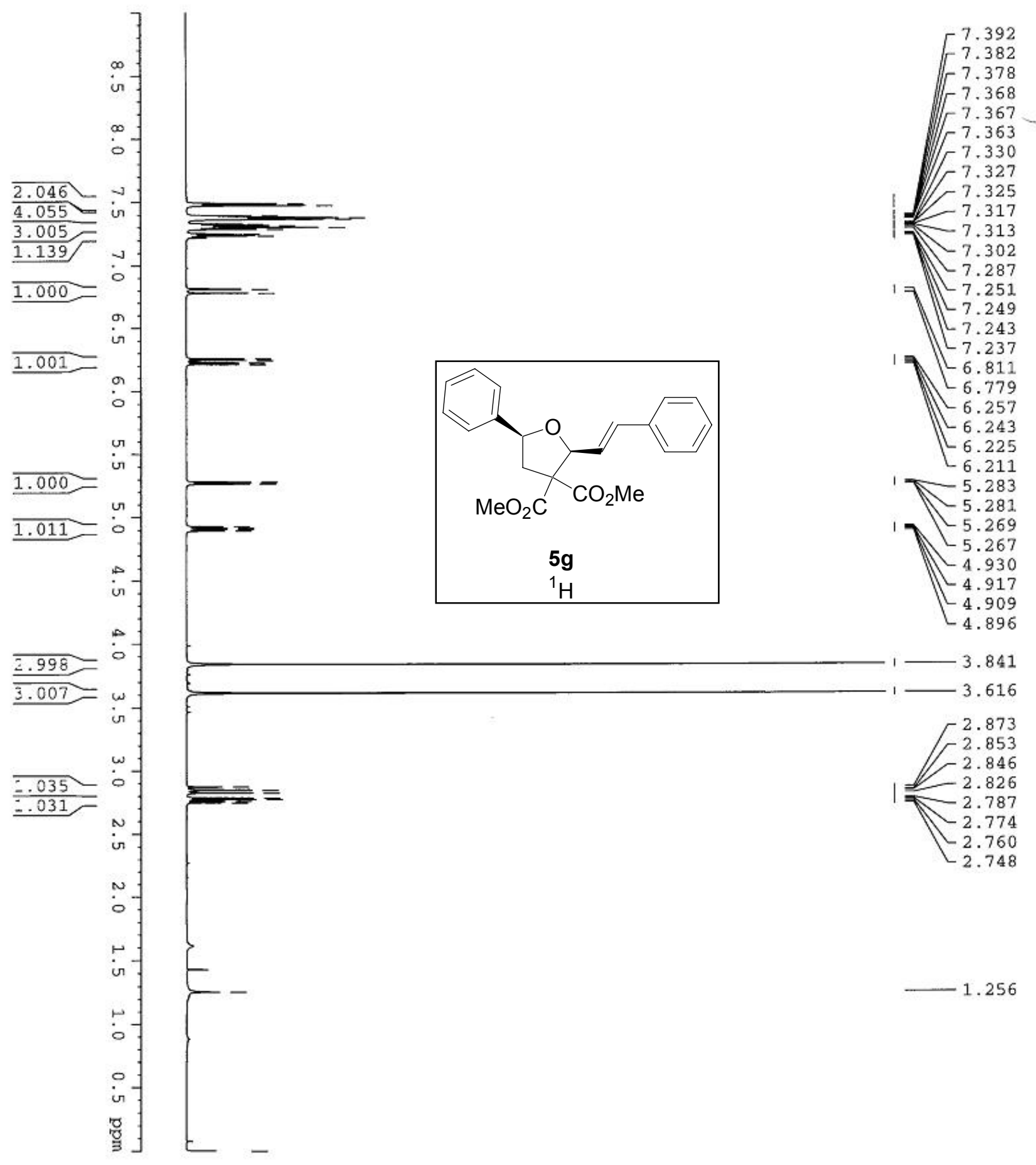




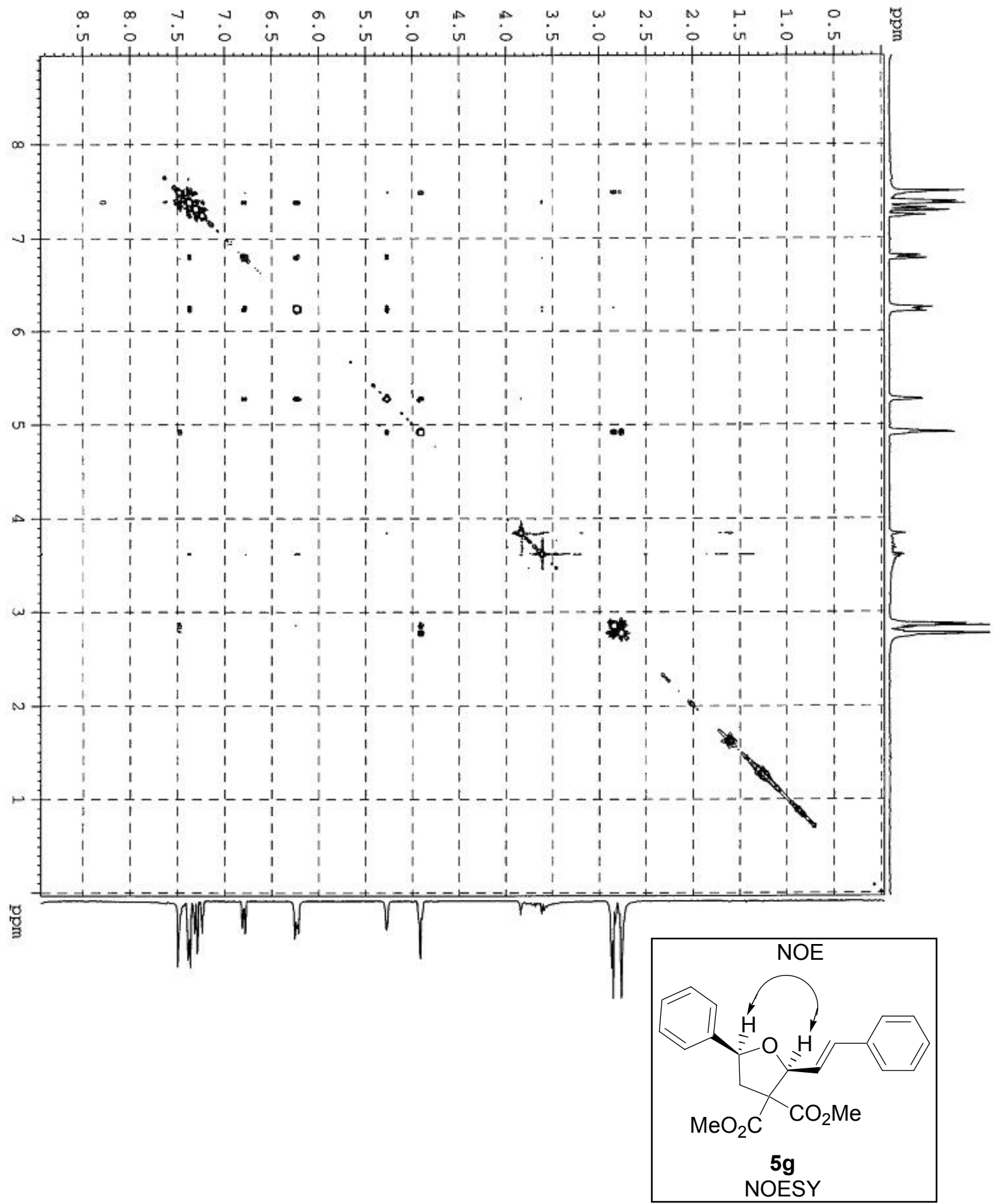









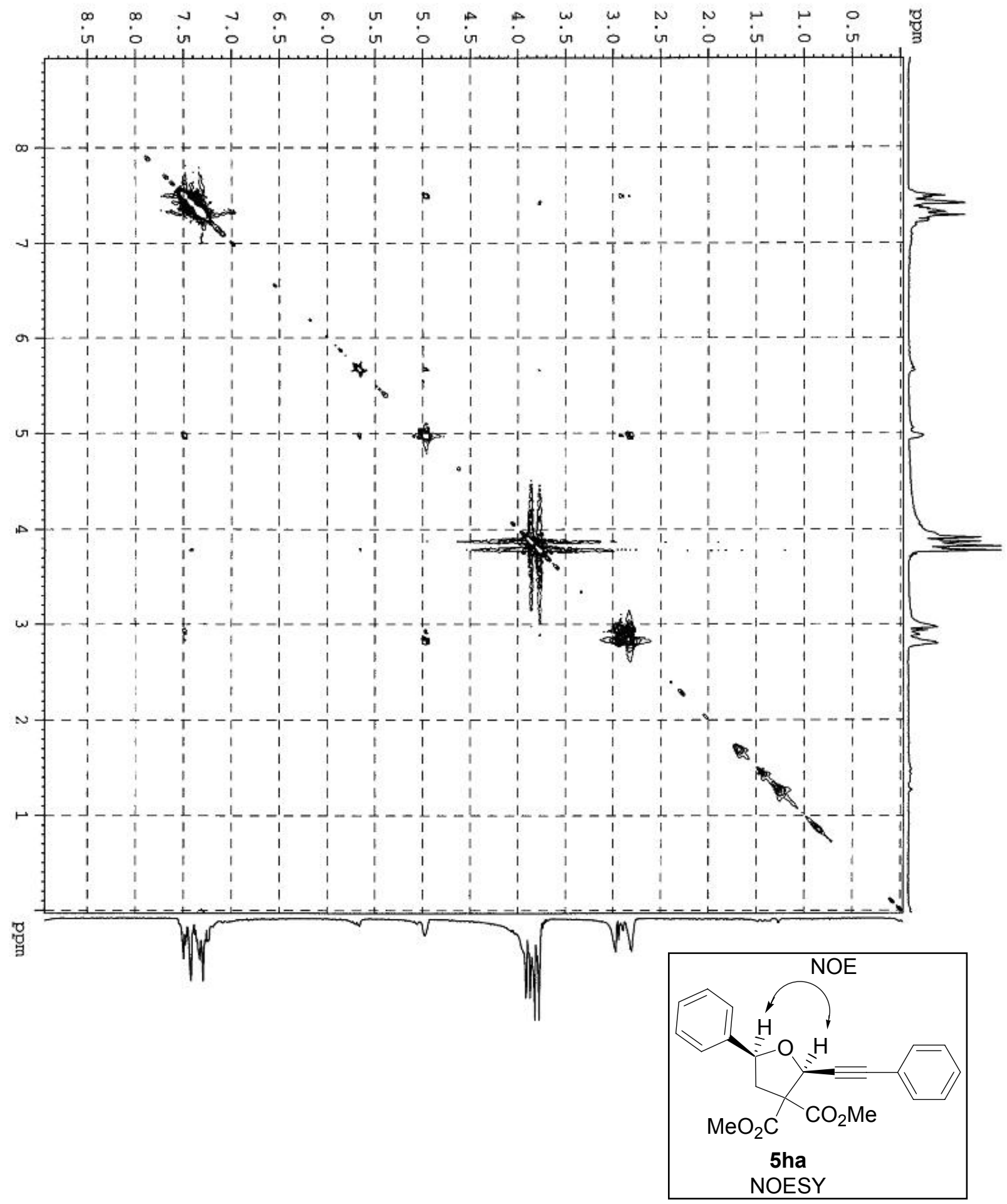




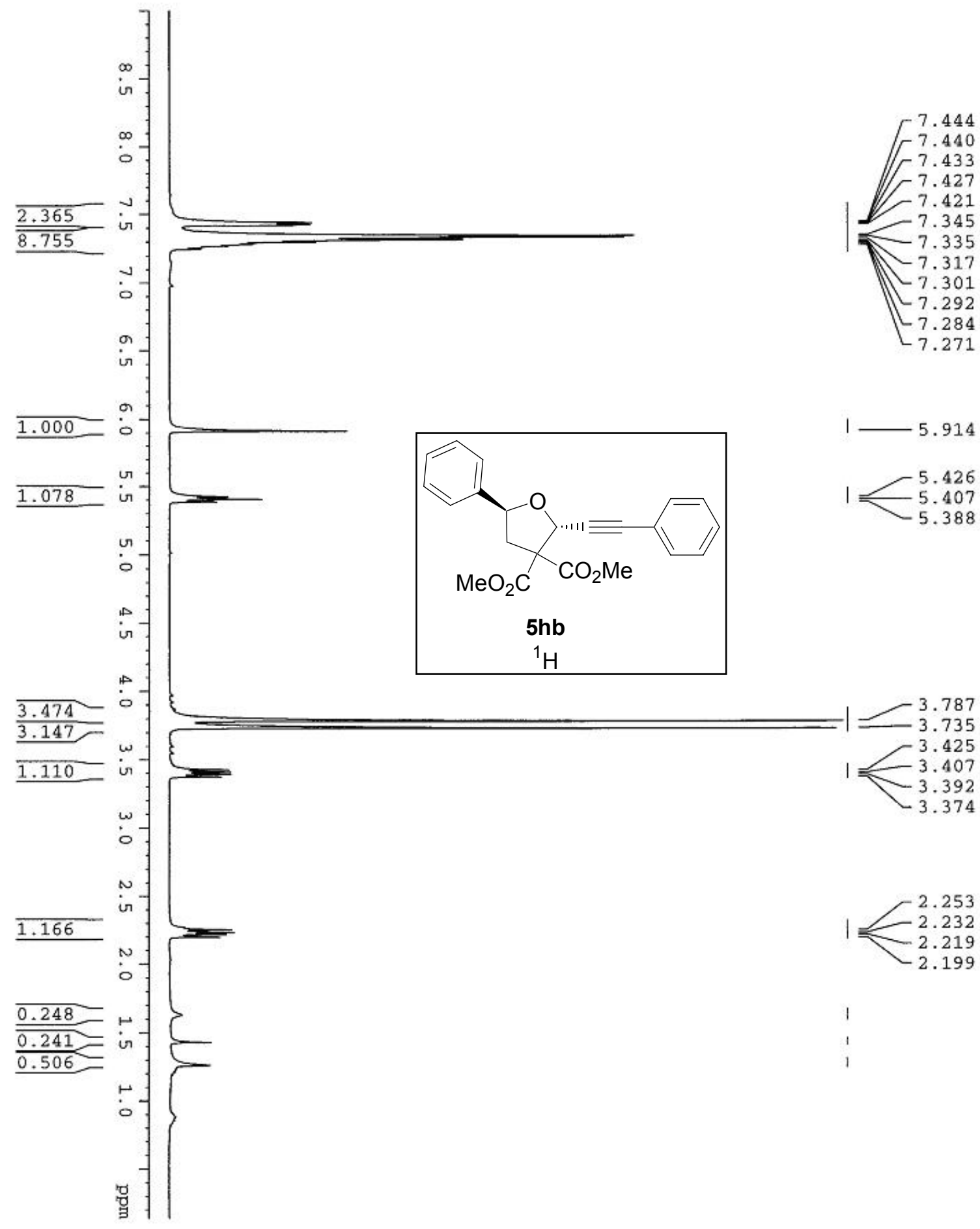




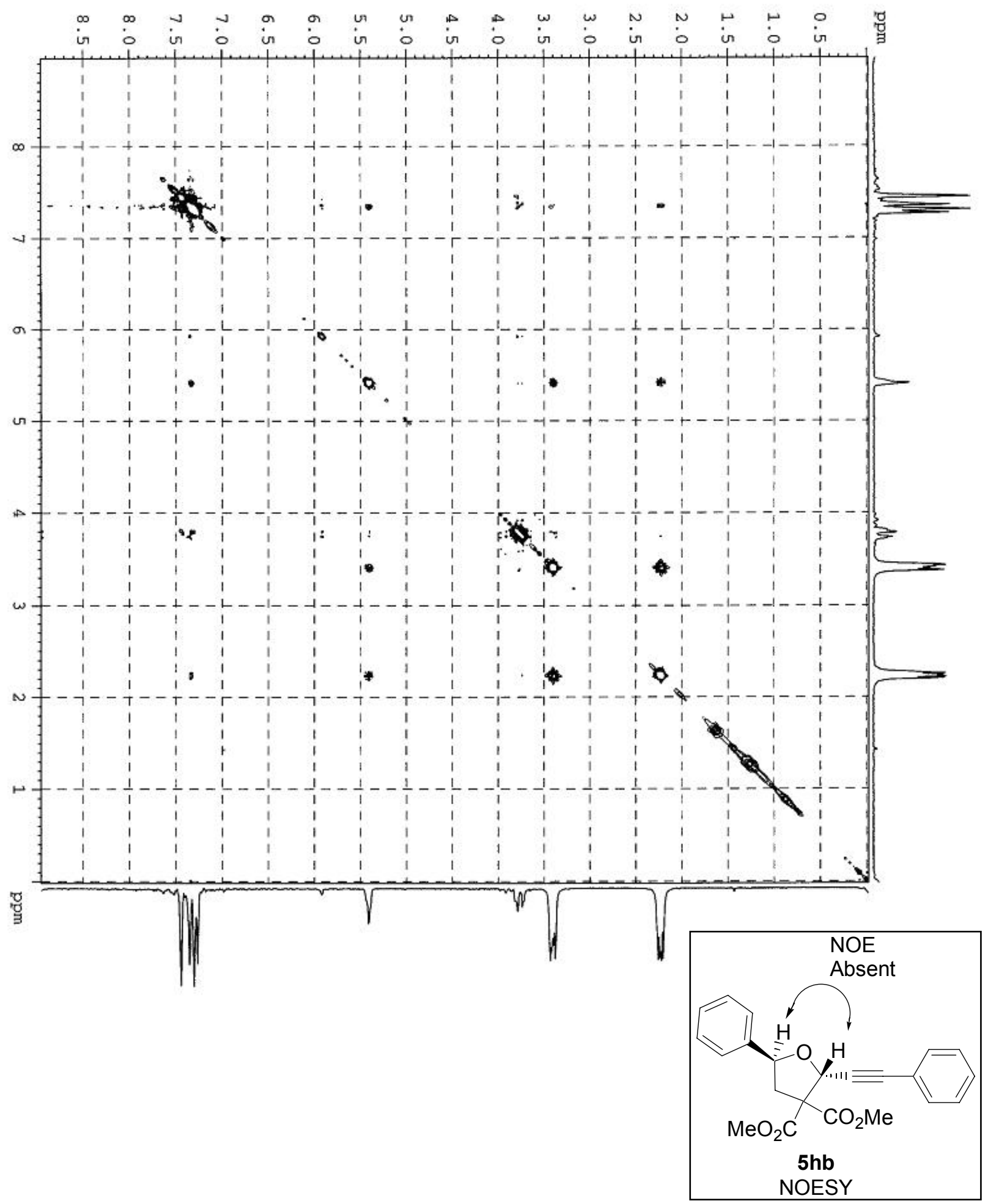




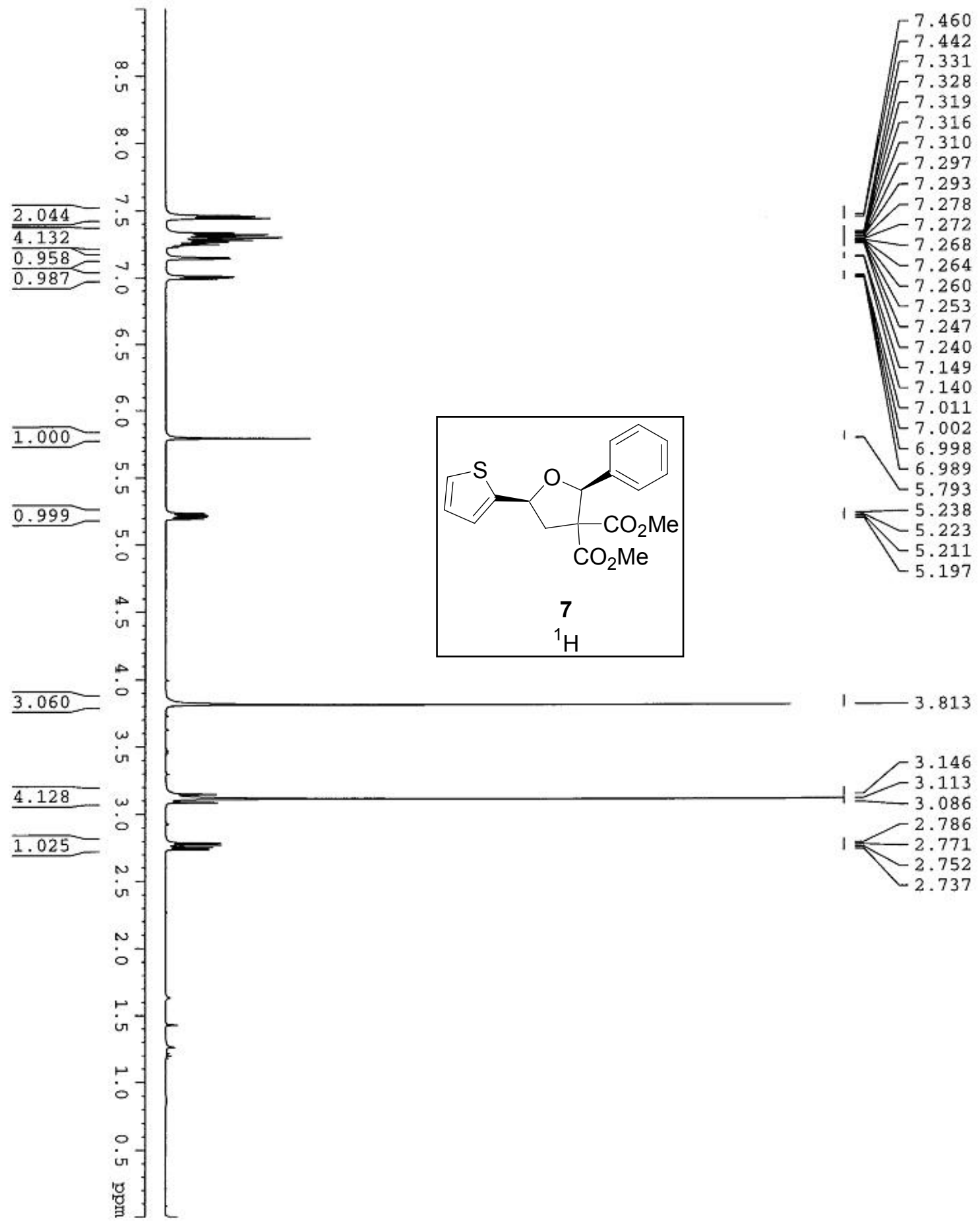




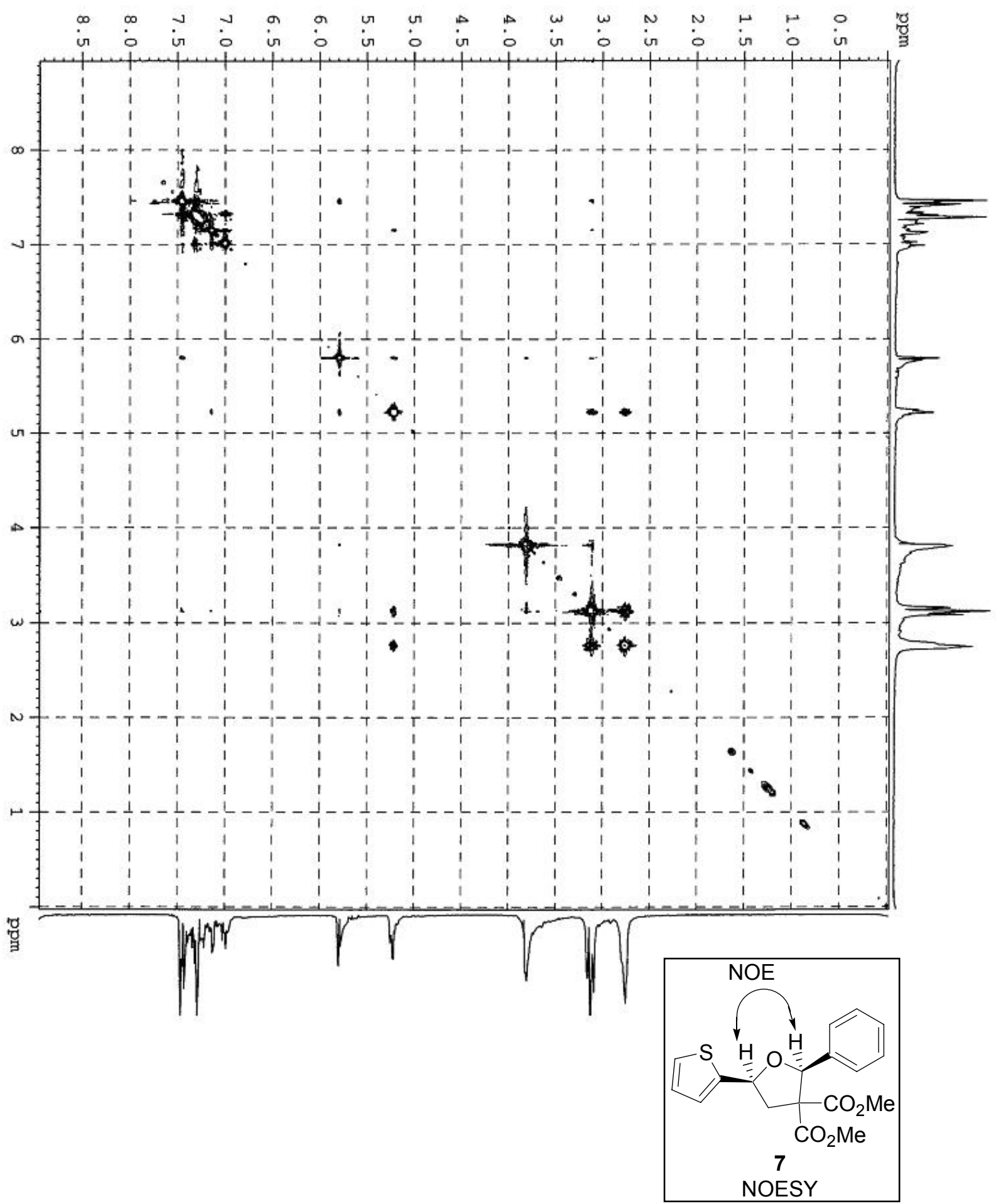

